JOURNAL OF THE

AMERICAN MATHEMATICAL SOCIETY

Volume 17, Number 2, Pages 473-497

S 0894-0347(03)00446-6

Article electronically published on November 25, 2003

\title{
ON THE SIZE OF $k$-FOLD SUM AND PRODUCT SETS OF INTEGERS
}

\author{
JEAN BOURGAIN AND MEI-CHU CHANG
}

\section{Statement of the RESult And outline of ARGUMENT}

We prove the following

Theorem. For all $b \in \mathbb{Z}_{+}$, there is $k=k(b) \in \mathbb{Z}_{+}$such that if $A \subset \mathbb{Z}$ is any finite set, with $|A|=N \geq 2$, then either

$$
|k A|=|\underbrace{A+\cdots+A}_{k}|>N^{b}
$$

or

$$
\left|A^{(k)}\right|=|\underbrace{A \times \cdots \times A}_{k}|>N^{b} .
$$

This result is one more contribution to a line of research initiated in the seminal paper E-S of Erdős and Szemerédi on sum and product sets. They conjectured that if $A \subset \mathbb{Z}$, with $|A|=N$, then

$$
|2 A|+\left|A^{(2)}\right|>c_{\varepsilon} N^{2-\varepsilon}, \quad \text { for all } \varepsilon>0,
$$

and more generally, for $k \geq 2$ an integer,

$$
|k A|+\left|A^{(k)}\right|>c_{\varepsilon} N^{k-\varepsilon}, \quad \text { for all } \varepsilon>0 .
$$

Already (i) is open. Recent advances were achieved by G. Elekes [E] and J. Solymosi [So] using the Szemerédi-Trotter theorem in incidence geometry. It was shown in E] that

$$
|2 A|+\left|A^{(2)}\right|>c N^{\frac{5}{4}},
$$

and in $\mathrm{So}$ the small further improvement

$$
|2 A|+\left|A^{(2)}\right|>c N^{\frac{14}{11}-\varepsilon}
$$

is given.

Again, using the Szemerédi-Trotter theorem, Elekes-Nathanson-Ruzsa showed that

$$
|k A| \cdot\left|A^{(k)}\right|>c|A|^{3-2^{1-k}} .
$$

(See $[\mathrm{E}-\mathrm{N}-\mathrm{R}]$.) The theorem proved in this paper answers affirmatively a problem posed in their paper and provides further progress towards (ii). This same issue was also brought up independently by S. Konjagin (private communication) and was

Received by the editors September 5, 2003.

1991 Mathematics Subject Classification. Primary 05A99.

(C)2003 American Mathematical Society 
motivated by questions related to Gauss-sums (see [B-K]). Some further refinements of the statement of the theorem appear in $\S 6$ (Remarks) at the end of this paper.

The general strategy of our proof bears resemblance with [Ch] in several ways. Thus we assume $\left|A^{(k)}\right|$ "small" and then prove that $|k A|$ has to be large. However, "smallness" of $|A \cdot A|$ in $[\mathrm{Ch}$ is the assumption

$$
|A \cdot A|<K|A|
$$

with $K$ a constant (a condition much too restrictive for our purpose).

If (1.4) holds, it is shown in [Ch] that

$$
|A+A|>c(K)|A|^{2}
$$

and more generally

$$
|h A|>c(K, h)|A|^{h} .
$$

Let us briefly recall the approach.

Consider the map given by prime factorization

$$
\begin{gathered}
\mathbb{Z}_{+} \longrightarrow \mathcal{R}=\prod_{p} \mathbb{Z}_{\geq 0}, \\
n=\prod_{p} p^{\alpha_{p}} \longrightarrow \alpha=\left(\alpha_{p}\right)_{p},
\end{gathered}
$$

where $p$ runs in the set $\mathcal{P}$ of primes.

The set $A$ is mapped to $\mathcal{A} \subset \mathcal{R}$ satisfying by (1.4)

$$
|2 \mathcal{A}|<K|\mathcal{A}| \text {. }
$$

Freiman's lemma then implies that $\operatorname{dim} \mathcal{A}<K$ (where "dim" refers to the dimension of the smallest vector space containing $\mathcal{A}$ ). Hence there is a subset $I \subset \mathcal{P},|I|<K$, such that the restriction $\pi_{I}$ is one-to-one restricted to $\mathcal{A}$. Harmonic analysis then implies that

$$
\lambda_{q}(A)<(C q)^{K}
$$

for an absolute constant $C$ and for all $q>2$. By $\lambda_{q}(A)$, we mean the $\Lambda_{q}$-constant of the finite set $A \subset \mathbb{Z}$, defined by

$$
\lambda_{q}(A)=\max \left\|\sum_{n \in A} c_{n} e^{2 \pi i n \theta}\right\|_{L^{q}(\mathbb{T})}
$$

where $\mathbb{T}=\mathbb{R} / \mathbb{Z}$ and the max is taken over all sequences $\left(c_{n}\right)_{n \in A}$ with $\left(\sum c_{n}^{2}\right)^{1 / 2} \leq$ 1. See $[\mathrm{Ru}$ and $[\mathrm{Ki}]$ for more details. As is $[\mathrm{Ch}]$, we will also make here crucial use of certain moment inequalities involving the $\lambda_{q}$-constant of certain specific sets of integers. More specifically, we will use the following general inequality, from which (1.7) is derived.

Proposition 1. (See [Ch].)

Let $\mathcal{P}_{0}=\left\{p_{1}, \ldots, p_{k}\right\}$ be a set of distinct primes and associate to each $\alpha=$ $\left(\alpha_{1}, \ldots, \alpha_{k}\right) \in\left(\mathbb{Z}_{\geq 0}\right)^{k}$ a trigonometric polynomial $F_{\alpha}$ on $\mathbb{T}$ such that

$$
(n, p)=1, \text { for all } n \in \operatorname{supp} \widehat{F}_{\alpha} \text { and for all } p \in \mathcal{P}_{0} \text {. }
$$

Then, for any moment $q \geq 2$

$$
\left\|\sum_{\alpha} F_{\alpha}\left(p_{1}^{\alpha_{1}} \cdots p_{k}^{\alpha_{k}} \theta\right)\right\|_{q}<(C q)^{k}\left(\sum\left\|F_{\alpha}\right\|_{q}^{2}\right)^{1 / 2} .
$$


Thus (1.7) follows from (1.9) taking $F_{\alpha}(\theta)=e^{2 \pi i \theta}$ and $\left\{p_{1}, \ldots, p_{k}\right\}=I \subset \mathcal{P}$.

Denote for $h \geq 2$,

$$
r_{h}(n ; A)=\left|\left\{\left(x_{1}, \ldots, x_{h}\right) \in A^{h} \mid n=x_{1}+\cdots+x_{h}\right\}\right| .
$$

A simple application of Parseval's identity gives

$$
\sum_{n \in h A} r_{h}(n ; A)^{2} \leq \lambda_{2 h}(A)^{2 h} \cdot|A|^{h}
$$

and using the Cauchy-Schwartz inequality on $\sum_{n \in h A} r_{h}(n ; A)$, it follows that

$$
|h A| \geq \frac{|A|^{h}}{\lambda_{2 h}(A)^{2 h}} .
$$

Thus we obtain (1.6) with

$$
c(K, h)>(C h)^{-2 h K} .
$$

Obviously, this statement has no interest unless $K \ll \log |A|$.

The main point in what follows is to be able to carry some of the preceding analysis under the much weaker assumption $K<|A|^{\varepsilon}, \varepsilon$ small. We will prove the following statement.

Proposition 2. Given $\gamma>0$ and $q>2$, there is a constant $\Lambda=\Lambda(\gamma, q)$ such that if $A \subset \mathbb{Z}$ is a finite set, $|A|=N,|A \cdot A|<K N$, then

$$
\lambda_{q}(A)<K^{\Lambda} N^{\gamma} \text {. }
$$

Thus fixing $q$, Proposition 2 provides already nontrivial information assuming $K<N^{\delta}$, with $\delta>0$ sufficiently small.

Assuming Proposition 2, let us derive the theorem. We may assume that $A \subset \mathbb{Z}_{+}$ to simplify the situation.

Fix $b$ and assume (1.2) fails for some large $k=2^{\ell}$ (to be specified). Hence, passing to $\mathcal{A}$,

$$
\begin{gathered}
|k \mathcal{A}|<N^{b} \\
\frac{\left|2^{\ell} \mathcal{A}\right|}{\left|2^{\ell-1} \mathcal{A}\right|} \frac{\left|2^{\ell-1} \mathcal{A}\right|}{\left|2^{\ell-2} \mathcal{A}\right|} \cdots \frac{|2 \mathcal{A}|}{|\mathcal{A}|}<N^{b-1},
\end{gathered}
$$

and we may find $k_{0}=2^{\ell_{0}}$ such that

$$
\frac{\left|2 k_{0} \mathcal{A}\right|}{\left|k_{0} \mathcal{A}\right|}<N^{\frac{b-1}{\ell}}
$$

Denote $\mathcal{B}=k_{0} \mathcal{A} \subset \mathcal{R}$ and $B=A^{\left(k_{0}\right)}$, the corresponding subset of $\mathbb{Z}_{+}$. Thus by

$$
|B \cdot B|<N^{\frac{b-1}{\ell}}|B| .
$$

Apply Proposition 2 to the set $B,|B| \equiv N_{0}, K=N^{\frac{b-1}{\ell}}$ with $\tau, \gamma$ specified later.

Hence from (1.11)

$$
\lambda_{q}(A) \leq \lambda_{q}(B)<N^{\frac{b-1}{\ell} \Lambda} N_{0}^{\gamma}<N^{\frac{b-1}{\ell} \Lambda+b \gamma} .
$$

Taking $q=2 h,(1.10)$ and (1.14) imply

$$
|h A|>N^{\left(1-2 \frac{b-1}{\ell} \Lambda-2 b \gamma\right) h} .
$$


Take $h=2 b<k, \gamma=\frac{1}{100 b}$. Recall that $\Lambda=\Lambda(\gamma, q)$, hence $\Lambda=\Lambda(b)$. Take $\ell=100 b \Lambda(b)$, so that $k=2^{\ell} \equiv k(b)$. Inequality (1.15) then clearly implies that

$$
|k A|>N^{b} .
$$

This proves the theorem.

Returning to Proposition 2, it will suffice to prove the following weaker version.

Proposition 2'. Given $\gamma>0, \tau>0$ and $q>2$ and $A$ as in Proposition 2, there is a subset $A^{\prime} \subset A$ satisfying

$$
\begin{aligned}
\left|A^{\prime}\right| & >N^{1-\tau}, \\
\lambda_{q}\left(A^{\prime}\right) & <K^{\Lambda} N^{\gamma},
\end{aligned}
$$

where $\Lambda=\Lambda(\tau, \gamma, q)$.

Proof of Proposition 2 assuming Proposition $2^{\prime}$. Denoting $\chi$ the indicator function, one has obviously

$$
\sum_{z \in \frac{A}{A^{\prime}}} \chi_{z A^{\prime}} \geq\left|A^{\prime}\right| \chi_{A}
$$

Let $A^{\prime}$ be the subset obtained in Proposition $2^{\prime}$. Then (1.18) is easily seen to imply

$$
\begin{aligned}
\left|A^{\prime}\right| \lambda_{q}(A) & \leq \sum_{z \in \frac{A}{A^{\prime}}} \lambda_{q}\left(z A^{\prime}\right) \\
& =\left|\frac{A}{A^{\prime}}\right| \lambda_{q}\left(A^{\prime}\right) \\
& \leq\left|\frac{A}{A}\right| K^{\Lambda} N^{\gamma} .
\end{aligned}
$$

If $\mathcal{A} \subset \mathcal{R}$ is the set introduced earlier, then application of Ruzsa's inequality on sum-difference sets gives

$$
\left|\frac{A}{A}\right|=|\mathcal{A}-\mathcal{A}| \leq K^{2}|\mathcal{A}|=K^{2} N
$$

Thus, by (1.16), (1.19) and (1.20), we have

$$
\lambda_{q}(A) \leq K^{\Lambda+2} N^{\tau+\gamma},
$$

where $\Lambda=\Lambda(\tau, \gamma, q)$. If we replace $\gamma$ by $\frac{\gamma}{2}$ and let $\tau=\frac{\gamma}{2}$, (1.11) follows.

The remainder of the paper is the proof of Proposition $2^{\prime}$ which will be rather tedious although elementary. The key statement is Proposition 3 below. This more technical result involves "graphs". We were unable to carry out our analysis by passing simply to subsets. As will be clear later on, the use of graphs indeed allows more flexibility in various constructions involving certain "regularizations". Trying to achieve them using subsets of $A$ is less economical and did not seem to provide us with the desired conclusions. These considerations are particularly relevant to Lemma 3.1 below (which is the base of the multi-scale analysis) and the difficulties encountered with its proof. 


\section{Reduction to Proposition 3 And PRELiminary EStimates}

Given subsets $A_{1}, A_{2}$ in an Abelian group and a symmetric graph $\mathcal{G} \subset A_{1} \times A_{2}$, define

$$
A_{1} \underset{\mathcal{G}}{+} A_{2}=\left\{x_{1}+x_{2} \mid\left(x_{1}, x_{2}\right) \in \mathcal{G}\right\} \text {. }
$$

For technical reason, we will prove a more general (and complicated) version of Proposition 2 (we consider subsets of $\mathcal{R}$, hence work in the additive setting).

Proposition 3. Given $\tau, \gamma>0$ and $q$, there is a constant $\Lambda=\Lambda(\tau, \gamma, q)$ such that the following holds. Let $\mathcal{P}_{0} \subset \mathcal{P}$ be a set of primes, and let $\mathcal{R}_{0}=\prod_{p \in \mathcal{P}_{0}} \mathbb{Z}_{\geq 0}$. Let $A_{1}, A_{2} \subset \mathcal{R}_{0}$ be finite with $\left|A_{i}\right|=N_{i}$ and let

$$
N=N_{1} N_{2} \text {. }
$$

If $\mathcal{G} \subset A_{1} \times A_{2}$ with

$$
|\mathcal{G}|>\delta N
$$

then there is $\mathcal{G}^{\prime} \subset \mathcal{G}$ satisfying

$$
\left|\mathcal{G}^{\prime}\right|>\delta^{\Lambda \log \log N} N^{1-\tau}
$$

and, moreover, for all $x \in \mathcal{R}_{0}$, the set $\mathcal{G}^{\prime}(x)=\left\{x^{\prime} \mid\left(x, x^{\prime}\right) \in \mathcal{G}^{\prime}\right.$ or $\left.\left(x^{\prime}, x\right) \in \mathcal{G}^{\prime}\right\}$ has the following property:

If $\left(F_{\alpha}\right)_{\alpha \in \mathcal{G}^{\prime}(x)}$ are arbitrary trigonometric polynomials such that $(n, p)=1$ for all $n \in \operatorname{supp} \widehat{F}_{\alpha}$ and for all $p \in \mathcal{P}_{0}$, then

$$
\left\|\sum_{\alpha} F_{\alpha}\left(\prod_{\mathcal{P}_{0}} p^{\alpha_{p}} \theta\right)\right\|_{q} \leq K(\mathcal{G})^{\Lambda} N^{\gamma}\left(\sum_{\alpha}\left\|F_{\alpha}\right\|_{q}^{2}\right)^{1 / 2}
$$

holds, where we denote

$$
K(\mathcal{G})=\frac{\left|A_{1}+\underset{\mathcal{G}}{+} A_{2}\right|}{\sqrt{N_{1} N_{2}}}
$$

Remark. If $A_{1}=A_{2}=A$ and $\mathcal{G}=A \times A$, then $K(\mathcal{G})$ is the doubling constant of $A$.

We will use the following

Notation. $M \sim N$ means that there are positive constants $c$ and $d$ such that $d N<M<c N$.

Proposition 3 implies Proposition 2'. Take $A_{1}=A_{2}=\mathcal{A} \subset \mathcal{R}, \mathcal{P}_{0}=\mathcal{P}$, and the full graph $\mathcal{G}=A_{1} \times A_{2} . \quad($ Note that $K(\mathcal{G})=K$, the doubling constant in Proposition 2.) Since by (2.1) $\mathcal{G}^{\prime} \subset \mathcal{A} \times \mathcal{A}$ satisfies

$$
\left|\mathcal{G}^{\prime}\right|>N^{2-3 \tau}
$$

there is $x \in \mathcal{A}$ such that $\left|\mathcal{G}^{\prime}(x)\right|>N^{1-3 \tau}$. Let $A^{\prime} \subset A$ be the set corresponding to $\mathcal{G}^{\prime}(x)$. Thus $\left|A^{\prime}\right|>N^{1-3 \tau}$ and, taking $F_{\alpha}(\theta)=c_{\alpha} e^{2 \pi i \theta}, c_{\alpha} \in \mathbb{R}$, statement $(2.2)$ clearly implies (1.17).

The proof of Proposition 3 will proceed in several stages. In this process, we consider pairs of functions

$$
\phi(N, \delta, K), \psi(N, \delta, K)
$$

such that under the assumptions of Proposition 3, there is $\mathcal{G}^{\prime} \subset \mathcal{G}$ satisfying

$$
\left|\mathcal{G}^{\prime}\right|>\phi(N, \delta, K(\mathcal{G})) \quad\left(N=N_{1} N_{2}\right)
$$


and (2.2) holds in the form

$$
\left\|\sum_{\alpha} F_{\alpha}\left(\prod p^{\alpha_{p}} \theta\right)\right\|_{q} \leq \psi(N, \delta, K(\mathcal{G}))\left(\sum_{\alpha}\left\|F_{\alpha}\right\|_{q}^{2}\right)^{1 / 2} .
$$

(We assume $q$ fixed.) We call such a pair of functions admissible.

The strategy then consists of getting better and better bounds on the functions $\phi(N, \delta, K), \psi(N, \delta, K)$ and eventually proving Proposition 3. Let us specify a first pair of admissible functions $\phi, \psi$ (see (2.13), (2.14)).

Assume $N_{1} \geq N_{2}$.

Obviously $\left|A_{1} \underset{\mathcal{G}}{+} A_{2}\right| \geq \frac{|\mathcal{G}|}{N_{2}} \geq \delta N_{1}$; hence

$$
\delta N_{1}<K(\mathcal{G})\left(N_{1} N_{2}\right)^{1 / 2} .
$$

Namely,

$$
N_{2}>\left(\frac{\delta}{K(\mathcal{G})}\right)^{2} N_{1}
$$

We may assume that $\mathcal{G} \subset A \times A$ is symmetric, where $A=A_{1} \cup A_{2}$, with $|A| \sim N_{1}$. Let

$$
K=K(\mathcal{G})
$$

We thus have

$$
\begin{aligned}
& |\mathcal{G}|>\delta N_{1} N_{2}>\frac{\delta^{3}}{K^{2}} N_{1}^{2}=\delta_{1} N_{1}^{2}, \\
& \left|A_{\mathcal{G}}^{+} A\right|=\left|A_{1} \underset{\mathcal{G}}{+} A_{2}\right|<K N_{1},
\end{aligned}
$$

where

$$
\delta_{1}=\frac{\delta^{3}}{K^{2}} .
$$

Applying Gower's version of the Balog-Szemerédi theorem (with powerlike estimate) (see [Go]), we may find a subset $A^{\prime} \subset A$ satisfying

$$
\begin{aligned}
& \left|A^{\prime}\right|>\delta^{\prime} N_{1}, \\
& \left|A^{\prime}-A^{\prime}\right|<K^{\prime} N_{1}, \\
& \left|\mathcal{G} \cap\left(A^{\prime} \times A^{\prime}\right)\right|>\delta^{\prime} N_{1}^{2},
\end{aligned}
$$

where

$$
\begin{gathered}
\delta^{\prime}>\left(\frac{\delta_{1}}{K}\right)^{C}>\left(\frac{\delta}{K}\right)^{C}, \\
K^{\prime}<\left(\frac{K}{\delta}\right)^{C}
\end{gathered}
$$

(here and in the sequel, notation $C$ as well as $c$ may refer to different constants).

By (2.6), (2.7) and Freiman's lemma, the dimension of the vector space spanned by $A^{\prime}$ is less than $\frac{K^{\prime}}{\delta^{\prime}}$. Thus there is a subset $I \subset \mathcal{P}_{0}$ such that

$$
|I|<\frac{K^{\prime}}{\delta^{\prime}}
$$

and the coordinate restriction $\pi_{I}: \mathcal{R}_{0} \rightarrow \prod_{p \in I} \mathbb{Z}_{\geq 0}$ is one-to-one when restricted to $A^{\prime}$. 
By (2.8), (2.9) let $\mathcal{G}^{\prime}=\mathcal{G} \cap\left(A^{\prime} \times A^{\prime}\right)$ satisfy

$$
\left|\mathcal{G}^{\prime}\right|>\left(\frac{\delta}{K}\right)^{C} N_{1} N_{2}
$$

Fix $x \in \mathcal{R}_{0}$ and consider $\mathcal{G}^{\prime}(x) \subset A^{\prime}$ and the trigonometric polynomials $F_{\alpha}, \alpha \in$ $\mathcal{G}^{\prime}(x)$, as in (2.2). Thus $(n, p)=1$, for all $n \in \operatorname{supp} \widehat{F}_{\alpha}$ and for all $p \in \mathcal{P}_{0}$. It follows from the preceding that $\alpha \in \mathcal{G}^{\prime}(x)$ is uniquely determined by e $\alpha^{\prime}=\pi_{I}(\alpha)$. Therefore clearly

$$
F_{\alpha}\left(\prod_{p \in \mathcal{P}_{0}} p^{\alpha_{p}} \theta\right)=F_{\alpha^{\prime}}\left(\prod_{p \in I} p^{\alpha_{p}} \theta\right)
$$

where $(n, p)=1$ for $n \in \operatorname{supp} \widehat{F}_{\alpha^{\prime}}$ and $p \in I$.

Thus Proposition 1 and (2.10) imply

$$
\begin{aligned}
\left\|\sum_{\alpha \in \mathcal{G}^{\prime}(x)} F_{\alpha}\left(\prod p^{\alpha_{p}} \theta\right)\right\|_{q} & =\left\|\sum_{\alpha} F_{\alpha^{\prime}}\left(\prod_{p \in I} p^{\alpha_{p}} \theta\right)\right\|_{q} \\
& \leq(C q)^{|I|}\left(\sum_{\alpha}\left\|F_{\alpha^{\prime}}\right\|_{q}^{2}\right)^{1 / 2} \\
& \leq(C q)^{\frac{K^{\prime}}{\delta^{\prime}}}\left(\sum_{\alpha}\left\|F_{\alpha}\right\|_{q}^{2}\right)^{1 / 2} .
\end{aligned}
$$

Hence, (2.11), (2.12), and (2.9) provide the following pair of admissible functions:

$$
\begin{aligned}
& \phi(N, \delta, K)=\left(\frac{\delta}{K}\right)^{C} N \\
& \psi(N, \delta, K)=\exp \left(\log q \cdot\left(\frac{K}{\delta}\right)^{C}\right)
\end{aligned}
$$

for some constant $C$.

Again the dependence of $\psi$ on $K$ is very poor, since it is a useless bound unless $K \ll \log N$.

The aim of what follows is to improve this dependence of $\psi$ on $K$.

\section{Proof of Proposition 3, Part I: The factorization}

The next statement is a recipe for converting pairs of admissible functions $\phi, \psi$. We will always assume

$\phi, \psi$ are increasing in $N$,

$\phi$ is increasing in $\delta$, decreasing in $K$,

$\psi$ increases in $K$

and

$$
\phi(N, \delta, K) \leq \frac{N}{M} \phi(M, \delta, K) \text { for } M \leq N
$$

Lemma 3.1. Let $\phi, \psi$ be admissible. Define

$$
\begin{aligned}
& \tilde{\phi}(N, \delta, K)=\min \phi\left(N^{\prime}, \delta^{\prime}, K^{\prime}\right) \cdot \phi\left(N^{\prime \prime}, \delta^{\prime \prime}, K^{\prime \prime}\right), \\
& \tilde{\psi}(N, \delta, K)=C q \max \psi\left(N^{\prime}, \delta^{\prime}, K^{\prime}\right) \cdot \psi\left(N^{\prime \prime}, \delta^{\prime \prime}, K^{\prime \prime}\right),
\end{aligned}
$$


where in (3.2), (3.3) the range of $N^{\prime}, N^{\prime \prime}, \delta^{\prime}, \delta^{\prime \prime}, K^{\prime}, K^{\prime \prime}$ are as follows:

$$
\begin{aligned}
& N \geq N^{\prime} N^{\prime \prime}>N\left(\frac{\delta}{\log K}\right)^{40}, \\
& N^{\prime}+N^{\prime \prime}<\left(\frac{K}{\delta}\right)^{15} N^{1 / 2}, \\
& \delta^{\prime} \cdot \delta^{\prime \prime}>\left(\log \frac{K}{\delta}\right)^{-6} \delta, \\
& K^{\prime} \cdot K^{\prime \prime}<\delta^{-14}(\log K)^{10} K .
\end{aligned}
$$

Then $\tilde{\phi}, \tilde{\psi}$ are also admissible.

This lemma is an essential ingredient in the proof of Proposition 3. In its proof, the role of graphs will become apparent.

Under the assumption of Proposition 3, we have $\mathcal{G} \subset A_{1} \times A_{2}$, for $A_{i} \subset \mathcal{R}_{0}=$ $\prod_{p \in \mathcal{P}_{0}} \mathbb{Z}_{\geq 0}$, with $\left|A_{i}\right|=N_{i}$, and

$$
\begin{aligned}
|\mathcal{G}| & >\delta N, \\
\left|A_{1}+{ }_{\mathcal{G}} A_{2}\right| & <K(\mathcal{G}) \sqrt{N},
\end{aligned}
$$

where $N=N_{1} N_{2}$.

The proof of Lemma 3.1 is in seven steps.

Step 1. For $i=1,2$, we reduce $A_{i}$ to $A_{i}^{\prime}$, with $\left|A_{i}^{\prime}\right|=N_{i}^{\prime}$ such that for any $B_{i} \subset A_{i}^{\prime}$,

$$
\begin{aligned}
\left|\mathcal{G} \cap\left(B_{1} \times A_{2}^{\prime}\right)\right| & >\frac{\delta}{4}\left|B_{1}\right| N_{2}^{\prime}, \\
\left|\mathcal{G} \cap\left(A_{1}^{\prime} \times B_{2}\right)\right| & >\frac{\delta}{4}\left|B_{2}\right| N_{1}^{\prime}
\end{aligned}
$$

and

$$
N_{i}^{\prime}>\frac{3 \delta}{4} N_{i}
$$

Moreover, the property

$$
\left|\mathcal{G} \cap\left(A_{1}^{\prime} \times A_{2}^{\prime}\right)^{c}\right| \leq \frac{\delta}{4}\left|\left(A_{1} \times A_{2}\right) \backslash\left(A_{1}^{\prime} \times A_{2}^{\prime}\right)\right|
$$

will hold.

Thus (3.11) implies (3.10), because

$$
N_{1}^{\prime} N_{2}^{\prime} \geq\left|\mathcal{G} \cap\left(A_{1}^{\prime} \times A_{2}^{\prime}\right)\right|>\delta N_{1} N_{2}-\frac{\delta}{4} N_{1} N_{2}=\frac{3 \delta}{4} N_{1} N_{2} .
$$

The construction is straightforward. Assume $A_{1}^{\prime} \times A_{2}^{\prime}$ fails (3.8). Thus

$$
\left|\mathcal{G} \cap\left(B_{1} \times A_{2}^{\prime}\right)\right| \leq \frac{\delta}{4}\left|B_{1}\right|\left|A_{2}^{\prime}\right|
$$

for some $B_{1} \subset A_{1}^{\prime}$. Define $A_{1}^{\prime \prime}=A_{1}^{\prime} \backslash B_{1}$. Then

$$
\begin{aligned}
\left|\mathcal{G} \cap\left(A_{1}^{\prime \prime} \times A_{2}^{\prime}\right)^{c}\right| & =\left|\mathcal{G} \cap\left(A_{1}^{\prime} \times A_{2}^{\prime}\right)^{c}\right|+\left|\mathcal{G} \cap\left(B_{1} \times A_{2}^{\prime}\right)\right| \\
& \leq \frac{\delta}{4}\left|\left(A_{1} \times A_{2}\right) \backslash\left(A_{1}^{\prime} \times A_{2}^{\prime}\right)\right|+\frac{\delta}{4}\left|B_{1}\right|\left|A_{2}^{\prime}\right| \\
& =\frac{\delta}{4}\left|\left(A_{1} \times A_{2}\right) \backslash\left(A_{1}^{\prime \prime} \times A_{2}^{\prime}\right)\right|
\end{aligned}
$$

and (3.11) remains valid. 
Continuing removing the bad set $B_{i},(3.11)$ ensures that the remaining set is still big enough, and the process gives the desired result.

Step 2. We decompose $\mathcal{P}_{0} \subset \mathcal{P}$ in disjoint sets $\mathcal{P}_{0}=\mathcal{P}_{1} \cup \mathcal{P}_{2}$.

The choice of this decomposition will matter only for condition (3.5).

We proceed as follows:

Enumerate $\mathcal{P}_{0}=\left\{p_{1}<p_{2}<\cdots<p_{t}\right\}$ which we identify with $\{1, \ldots, t\}$.

For $t^{\prime} \leq t$, consider the decreasing functions $(i=1,2)$

$$
n_{i}\left(t^{\prime}\right)=\max _{\left(x_{1}, \ldots, x_{t^{\prime}}\right) \in \mathbb{Z}^{\prime}}\left|A_{i}\left(x_{1}, \ldots, x_{t^{\prime}}\right)\right|,
$$

where $A_{i}\left(x_{1}, \ldots, x_{t^{\prime}}\right)=\left\{\left(x_{t^{\prime}+1}, \ldots, x_{t}\right) \mid\left(x_{1}, \ldots, x_{t}\right) \in A_{i}\right\}$.

We take $t^{\prime}$ such that

$$
\left\{\begin{array}{l}
n_{1}\left(t^{\prime}\right)+n_{2}\left(t^{\prime}\right) \geq\left(N_{1} N_{2}\right)^{1 / 4} \\
n_{1}\left(t^{\prime}+1\right)+n_{2}\left(t^{\prime}+1\right) \leq\left(N_{1} N_{2}\right)^{1 / 4} .
\end{array}\right.
$$

We assume $n_{1}\left(t^{\prime}\right) \geq n_{2}\left(t^{\prime}\right)$. Thus

$$
n_{1}\left(t^{\prime}\right) \geq \frac{1}{2} N^{1 / 4} .
$$

Then decompose then $\mathcal{P}_{0}=\mathcal{P}_{1} \cup \mathcal{P}_{2}$ where $\mathcal{P}_{1}=\left\{p_{1}, \ldots, p_{t^{\prime}}\right\}$.

Step 3. Let $\mathcal{R}_{i}=\prod_{p \in \mathcal{P}_{i}} \mathbb{Z}_{\geq 0}$ with $\mathcal{R}_{0}=\mathcal{R}_{1} \times \mathcal{R}_{2}$ corresponding to the decomposition in Step 2, and let $\pi_{1}: \mathcal{R}_{0} \rightarrow \mathcal{R}_{1}$ be the projection to the first $t^{\prime}$ coordinates. Denote

$$
\bar{x}=\left(x_{1}, \ldots, x_{t^{\prime}}\right) .
$$

We construct a set $\overline{\bar{A}}_{2} \subset A_{2}^{\prime}$ such that for all $\bar{x} \in \pi_{1}\left(\overline{\bar{A}}_{2}\right)$, we have $\left|\overline{\bar{A}}_{2}(\bar{x})\right| \sim m_{2}>$ $c \delta^{5} K^{-2} N^{1 / 4}, M_{2} \equiv\left|\pi_{1}\left(\overline{\bar{A}}_{2}\right)\right| \sim \frac{\left|\overline{\bar{A}}_{2}\right|}{m_{2}}<C \delta^{-5} K^{2} \frac{N_{2}}{N^{1 / 4}}$, and $\overline{\bar{N}}_{2} \equiv\left|\overline{\bar{A}}_{2}\right|>c \frac{\delta^{3}}{\log \frac{K}{\delta}} N_{2}$.

Choose $\bar{x} \in \pi_{1}\left(A_{1}^{\prime}\right)$ such that

$$
\left|A_{1}^{\prime}(\bar{x})\right|=n_{1}\left(t^{\prime}\right)
$$

It follows from (3.8) that

$$
\left|\mathcal{G} \cap\left[\left(\{\bar{x}\} \times A_{1}^{\prime}(\bar{x})\right) \times A_{2}^{\prime}\right]\right|>\frac{\delta}{4} n_{1}\left(t^{\prime}\right) N_{2}^{\prime}
$$

and hence there is a subset $A_{2}^{\prime \prime} \subset A_{2}^{\prime}$ such that by Fact 1 below,

$$
N_{2}^{\prime \prime}=\left|A_{2}^{\prime \prime}\right|>\frac{\delta}{8} N_{2}^{\prime}
$$

and for $z \in A_{2}^{\prime \prime}$

$$
\left|\mathcal{G} \cap\left[\left(\{\bar{x}\} \times A_{1}^{\prime}(\bar{x})\right) \times\{z\}\right]\right|>\frac{\delta}{8} n_{1}\left(t^{\prime}\right) .
$$

Fact 1. Let $|E| \leq e$ and $|F| \leq f$. If $|\mathcal{G} \cap(E \times F)|>\alpha e f$, then there exists $F^{\prime} \subset F$ with $\left|F^{\prime}\right|>\frac{\alpha}{2} f$, such that for any $z \in F^{\prime},|\mathcal{G} \cap(E \times\{z\})|>\frac{\alpha}{2} e$. 
From (2.5) and (3.16), we clearly get

$$
\begin{aligned}
\frac{K^{2}}{\delta} N_{2} \geq K \sqrt{N_{1} N_{2}} & =\left|A_{1} \underset{\mathcal{G}}{+} A_{2}\right| \\
& \geq\left|\left(\{\bar{x}\} \times A_{1}^{\prime}(\bar{x})\right) \underset{\mathcal{G}}{+} A_{2}^{\prime \prime}\right|>\frac{\delta}{8}\left|\pi_{1}\left(A_{2}^{\prime \prime}\right)\right| \cdot n_{1}\left(t^{\prime}\right) .
\end{aligned}
$$

In particular, $\frac{\left|A_{2}^{\prime \prime}\right|}{\left|\pi_{1}\left(A_{2}^{\prime \prime}\right)\right|} \geq \frac{3}{256} \frac{\delta^{4}}{K^{2}} n_{1}\left(t^{\prime}\right)$.

Let $\bar{A}_{2} \subset A_{2}^{\prime \prime}$ such that the fibers over $\bar{x}$ have size at least $\frac{\delta^{5} n_{1}\left(t^{\prime}\right)}{10^{4} K^{2}}$. Thus

$$
\bar{A}_{2}=\bigcup_{\left|A_{2}^{\prime \prime}(\bar{x})\right|>10^{-4} \delta^{5} K^{-2} n_{1}\left(t^{\prime}\right)}\left(\{\bar{x}\} \times A_{2}^{\prime \prime}(\bar{x})\right) .
$$

It follows from (3.17) that

$$
\left|A_{2}^{\prime \prime} \backslash \bar{A}_{2}\right| \leq\left|\pi_{1}\left(A_{2}^{\prime \prime}\right)\right| 10^{-4} \delta^{5} K^{-2} n_{1}\left(t^{\prime}\right)<\delta^{3} 10^{-3} N_{2}<\frac{\delta}{10} N_{2}^{\prime \prime} .
$$

The last inequality is true by (3.10) and (3.15).

Since by (3.9)

$$
\left|\mathcal{G} \cap\left(A_{1}^{\prime} \times A_{2}^{\prime \prime}\right)\right|>\frac{\delta}{4} N_{1}^{\prime} N_{2}^{\prime \prime},
$$

it follows from (3.18) that

$$
\left|\mathcal{G} \cap\left(A_{1}^{\prime} \times \bar{A}_{2}\right)\right|>\frac{\delta}{4} N_{1}^{\prime} N_{2}^{\prime \prime}-\frac{\delta}{10} N_{1}^{\prime} N_{2}^{\prime \prime}>\frac{\delta}{10} N_{1}^{\prime} N_{2}^{\prime \prime} .
$$

Since $\left|A_{2}^{\prime \prime}(\bar{x})\right| \leq n_{2}\left(t^{\prime}\right) \leq n_{1}\left(t^{\prime}\right)$, we may specify $m_{2}$ and $\overline{\bar{A}}_{2}$ as follows:

$$
10^{-4} \delta^{5} K^{-2} n_{1}\left(t^{\prime}\right)<m_{2}<n_{1}\left(t^{\prime}\right),
$$

and

$$
A_{2}^{\prime} \supset A_{2}^{\prime \prime} \supset \bar{A}_{2} \supset \overline{\bar{A}}_{2}=\bigcup_{\left|A_{2}^{\prime \prime}(\bar{x})\right| \sim m_{2}}\left(\{\bar{x}\} \times A_{2}^{\prime \prime}(\bar{x})\right)
$$

such that

$$
\left|\mathcal{G} \cap\left(A_{1}^{\prime} \times \overline{\bar{A}}_{2}\right)\right|>c \frac{\delta}{\log \frac{K}{\delta}} N_{1}^{\prime} N_{2}^{\prime \prime} .
$$

Thus $\overline{\bar{N}}_{2}=\left|\overline{\bar{A}}_{2}\right|$ satisfies

$$
\overline{\bar{N}}_{2}>c \frac{\delta}{\log \frac{K}{\delta}} N_{2}^{\prime \prime}>c \frac{\delta^{3}}{\log \frac{K}{\delta}} N_{2} .
$$

The set $\overline{\bar{A}}_{2}$ has a "regular" structure with respect to the decomposition $\mathcal{R}_{0}=$ $\mathcal{R}_{1} \times \mathcal{R}_{2}$ such that for all $\bar{x} \in \pi_{1}\left(\overline{\bar{A}}_{2}\right)$ we have $\left|\overline{\bar{A}}_{2}(\bar{x})\right| \sim m_{2}$. In particular, denoting $M_{2}=\left|\pi_{1}\left(\overline{\bar{A}}_{2}\right)\right|$, we have

$$
\overline{\bar{N}}_{2} \sim M_{2} \cdot m_{2} .
$$

By (3.20) and (3.13)

$$
m_{2}>c \delta^{5} K^{-2} N^{1 / 4}
$$

and

$$
M_{2}<C \delta^{-5} K^{2} \frac{N_{2}}{N^{1 / 4}} .
$$


Step 4. Regularization of $A_{1}^{\prime}$. We construct a set $\overline{\bar{A}}_{1} \subset A_{1}^{\prime}$ such that for any $\bar{x} \in \pi_{1}\left(\overline{\bar{A}}_{1}\right)$, we have $\left|\overline{\bar{A}}_{1}(\bar{x})\right| \sim m_{1}>c \delta^{10} K^{-5} N^{1 / 4}, M_{1} \equiv\left|\pi_{1}\left(\overline{\bar{A}}_{1}\right)\right| \sim \frac{\left|\overline{\bar{A}}_{1}\right|}{m_{1}}<$ $C \delta^{-10} K^{5} \frac{N_{1}}{N^{1 / 4}}, \quad \overline{\bar{N}}_{1}=\left|\overline{\bar{A}}_{1}\right|>c \frac{\delta^{2}}{\left(\log \frac{K}{\delta}\right)^{2}} N_{1}$, and $\left|\mathcal{G} \cap\left(\overline{\bar{A}}_{1} \times \overline{\bar{A}}_{2}\right)\right|>c \frac{\delta}{\left(\log \frac{K}{\delta}\right)^{2}} \overline{\bar{N}}_{1} \overline{\bar{N}}_{2}$.

Claim. Let $\tilde{A}_{1} \subset A_{1}^{\prime}$. If

$$
\left|\mathcal{G} \cap\left(\tilde{A}_{1} \times \overline{\bar{A}}_{2}\right)\right| \sim\left|\mathcal{G} \cap\left(A_{1}^{\prime} \times \overline{\bar{A}}_{2}\right)\right|,
$$

then

$$
m \equiv \max _{\bar{x} \in \pi_{1}\left(\tilde{A}_{1}\right)}\left|\tilde{A}_{1}(\bar{x})\right|>c \frac{\delta^{4}}{\left(\log \frac{K}{\delta}\right)^{2}} K^{-2} m_{2}
$$

Proof of Claim. From (3.25), (3.22) and the regular structure of $\overline{\bar{A}}_{2}$, there is $\bar{x} \in$ $\pi_{1}\left(\overline{\bar{A}}_{2}\right)$ such that

$$
\left|\mathcal{G} \cap\left(\tilde{A}_{1} \times\left(\{\bar{x}\} \times \overline{\bar{A}}_{2}(\bar{x})\right)\right)\right|>c \frac{\delta}{\log \frac{K}{\delta}} N_{1}^{\prime} m_{2} .
$$

Hence by Fact 1 , there is a subset $A_{1}^{\prime \prime} \subset \tilde{A}_{1}$ satisfying

$$
\left|A_{1}^{\prime \prime}\right|>c \frac{\delta}{\log \frac{K}{\delta}} N_{1}^{\prime}
$$

and for any $z \in A_{1}^{\prime \prime}$

$$
\left|\mathcal{G} \cap\left(\{z\} \times\left(\{\bar{x}\} \times \overline{\bar{A}}_{2}(\bar{x})\right)\right)\right|>c \frac{\delta}{\log \frac{K}{\delta}} m_{2} .
$$

As in Step 3, (3.17), write

$$
\begin{aligned}
\frac{K^{2}}{\delta} N_{1} \geq K \sqrt{N_{1} N_{2}} \geq\left|A_{1} \underset{\mathcal{G}}{+} A_{2}\right| & \geq\left|A_{1}^{\prime \prime} \underset{\mathcal{G}}{+}\left(\{\bar{x}\} \times \overline{\bar{A}}_{2}(\bar{x})\right)\right| \\
& >c\left|\pi_{1}\left(A_{1}^{\prime \prime}\right)\right| \frac{\delta}{\log \frac{K}{\delta}} m_{2} \\
& >c \frac{\left|A_{1}^{\prime \prime}\right|}{m} \frac{\delta}{\log \frac{K}{\delta}} m_{2} \\
& >c \frac{\delta^{3}}{\left(\log \frac{K}{\delta}\right)^{2}} \frac{m_{2}}{m} N_{1} .
\end{aligned}
$$

The last two inequalities are true by the definition of $m$ in (3.26) and (3.27), (3.10). Hence

$$
m>c \frac{\delta^{4}}{\left(\log \frac{K}{\delta}\right)^{2}} K^{-2} m_{2}
$$

Clearly, the bound in (3.26) is bigger than $\delta^{5} K^{-3} m_{2}$. Therefore, in (3.22) we may replace $A_{1}^{\prime}$ by $\bar{A}_{1}$ defined as follows:

$$
A_{1}^{\prime} \supset \bar{A}_{1}=\bigcup_{\left|A_{1}^{\prime}(\bar{x})\right|>\delta^{5} K^{-3} m_{2}}\left(\{\bar{x}\} \times A_{1}^{\prime}(\bar{x})\right) .
$$


Thus, applying the claim to $A_{1}^{\prime}-\bar{A}_{1}$, we have that

$$
\left|\mathcal{G} \cap\left(\bar{A}_{1} \times \overline{\bar{A}}_{2}\right)\right|>c \frac{\delta}{\log \frac{K}{\delta}} N_{1}^{\prime} N_{2}^{\prime \prime} .
$$

Recalling (3.20), for $\bar{x} \in \pi_{1}\left(\bar{A}_{1}\right)$

$$
\delta^{5} K^{-3} m_{2}<\left|\bar{A}_{1}(\bar{x})\right| \leq n_{1}\left(t^{\prime}\right)<C \delta^{-5} K^{2} m_{2} .
$$

Keeping (3.22) and (3.25) in mind, we may thus again specify

$$
\delta^{5} K^{-3} m_{2}<m_{1}<C \delta^{-5} K^{2} m_{2}
$$

such that the regular set $\overline{\bar{A}}_{1}$ defined as

$$
A_{1}^{\prime} \supset \bar{A}_{1} \supset \overline{\bar{A}}_{1}=\bigcup_{\left|\bar{A}_{1}(\bar{x})\right| \sim m_{1}}\left(\{\bar{x}\} \times \bar{A}_{1}(\bar{x})\right)
$$

will satisfy

$$
\left|\mathcal{G} \cap\left(\overline{\bar{A}}_{1} \times \overline{\bar{A}}_{2}\right)\right|>c \frac{\delta}{\left(\log \frac{K}{\delta}\right)^{2}} N_{1}^{\prime} N_{2}^{\prime \prime} .
$$

Denoting $\overline{\bar{N}}_{1}=\left|\overline{\bar{A}}_{1}\right|, M_{1}=\left|\pi_{1}\left(\overline{\bar{A}}_{1}\right)\right|$, we have $\overline{\bar{N}}_{1} \sim M_{1} m_{1}$. On the other hand, (3.31), (3.10) and the fact that $\overline{\bar{A}}_{i} \subset A_{i}^{\prime \prime}$ give

$$
\overline{\bar{N}}_{1}>c \frac{\delta^{2}}{\left(\log \frac{K}{\delta}\right)^{2}} N_{1}
$$

and

$$
\left|\mathcal{G} \cap\left(\overline{\bar{A}}_{1} \times \overline{\bar{A}}_{2}\right)\right|>c \frac{\delta}{\left(\log \frac{K}{\delta}\right)^{2}} \overline{\bar{N}}_{1} \overline{\bar{N}}_{2} .
$$

It follows from (3.24) and (3.29) that

$$
\begin{aligned}
& m_{1}>c \delta^{10} K^{-5} N^{1 / 4}, \\
& M_{1}<C \delta^{-10} K^{5} \frac{N_{1}}{N^{1 / 4}} .
\end{aligned}
$$

Thus at this stage we have regularized both $A_{1}, A_{2}$ with respect to the decomposition $\mathcal{P}_{0}=\mathcal{P}_{1} \cup \mathcal{P}_{2}$.

For simplicity, we redenote $\overline{\bar{A}}_{1}, \overline{\bar{A}}_{2}$ by $A_{1}, A_{2}$ whose cardinalities $\overline{\bar{N}}_{i} \sim m_{i} M_{i}$ satisfy (3.23) and (3.32).

For $\bar{x}_{1}, \bar{x}_{2} \in \mathcal{R}_{1}$, let $\mathcal{G}_{\bar{x}_{1}, \bar{x}_{2}} \subset \mathcal{R}_{2} \times \mathcal{R}_{2}$ be the fiber of $\mathcal{G}$ over $\left(\bar{x}_{1}, \bar{x}_{2}\right)$,

$$
\mathcal{G}_{\bar{x}_{1}, \bar{x}_{2}}=\left\{\left(\bar{y}_{1}, \bar{y}_{2}\right) \in A_{1}\left(\bar{x}_{1}\right) \times A_{2}\left(\bar{x}_{2}\right) \mid\left(\left(\bar{x}_{1}, \bar{y}_{1}\right),\left(\bar{x}_{2}, \bar{y}_{2}\right)\right) \in \mathcal{G}\right\} \subset \mathcal{R}_{2} \times \mathcal{R}_{2} .
$$

Step 5. Regularization of the graph. We construct $\mathcal{G}_{1,0} \subset \pi_{1}\left(A_{1}\right) \times \pi_{1}\left(A_{2}\right) \subset \mathcal{R}_{1} \times$ $\mathcal{R}_{1}$ with $\left|\mathcal{G}_{1,0}\right|>\delta_{0} M_{1} M_{2}$, such that $\forall\left(\bar{x}_{1}, \bar{x}_{2}\right) \in \mathcal{G}_{1,0}$, we have $\left|A_{1}\left(\bar{x}_{1}\right){ }_{\mathcal{G}_{\bar{x}_{1}, \bar{x}_{2}}} A_{2}\left(\bar{x}_{2}\right)\right|$ $\sim L \sqrt{m_{1} m_{2}}$ with $L<L_{0}$, and $\left|\mathcal{G}_{\bar{x}_{1}, \bar{x}_{2}}\right| \sim \delta_{1} m_{1} m_{2}$, where $\mathcal{G}_{\bar{x}_{1}, \bar{x}_{2}}$ is the fiber over $\left(\bar{x}_{1}, \bar{x}_{2}\right)$, and $\delta_{0}, \delta_{1}$ and $L_{0}$ satisfy (3.44), (3.37) and (3.42), respectively.

It follows from (3.33) that we may restrict $\mathcal{G}$ to $\mathcal{G}_{1} \times\left(\mathcal{R}_{2} \times \mathcal{R}_{2}\right)$, where

$$
\mathcal{G}_{1}=\left\{\left(\bar{x}_{1}, \bar{x}_{2}\right) \in \pi_{1}\left(A_{1}\right) \times \pi_{1}\left(A_{2}\right)|| \mathcal{G}_{\bar{x}_{1}, \bar{x}_{2}} \mid>c \frac{\delta}{\left(\log \frac{K}{\delta}\right)^{2}} m_{1} m_{2}\right\} .
$$


Thus

and by $(3.33)$

$$
m_{1} m_{2} \geq\left|\mathcal{G}_{\bar{x}_{1}, \bar{x}_{2}}\right|>c \frac{\delta}{\left(\log \frac{K}{\delta}\right)^{2}} m_{1} m_{2}, \text { for }\left(\bar{x}_{1}, \bar{x}_{2}\right) \in \mathcal{G}_{1}
$$

$$
\sum_{\left(\bar{x}_{1}, \bar{x}_{2}\right) \in \mathcal{G}_{1}}\left|\mathcal{G}_{\bar{x}_{1}, \bar{x}_{2}}\right|>c \frac{\delta}{\left(\log \frac{K}{\delta}\right)^{2}} \overline{\bar{N}}_{1} \overline{\bar{N}}_{2} .
$$

We may thus specify $\delta_{1}$ :

$$
1>\delta_{1}>c \frac{\delta}{\left(\log \frac{K}{\delta}\right)^{2}}
$$

such that if

$$
\mathcal{G}_{1}^{\prime}=\left\{\left(\bar{x}_{1}, \bar{x}_{2}\right) \in \mathcal{G}_{1}|| \mathcal{G}_{\bar{x}_{1}, \bar{x}_{2}} \mid \sim \delta_{1} m_{1} m_{2}\right\}
$$

then we have

$$
\sum_{\left(\bar{x}_{1}, \bar{x}_{2}\right) \in \mathcal{G}_{1}^{\prime}}\left|\mathcal{G}_{\bar{x}_{1}, \bar{x}_{2}}\right|>c \frac{\delta}{\left(\log \frac{K}{\delta}\right)^{3}} \overline{\bar{N}}_{1} \overline{\bar{N}}_{2}
$$

Hence

$$
\left|\mathcal{G}_{1}^{\prime}\right|>c \frac{\delta}{\delta_{1}\left(\log \frac{K}{\delta}\right)^{3}} M_{1} M_{2},
$$

which is bigger than $\frac{\delta}{\left(\log \frac{K}{\delta}\right)^{3}} M_{1} M_{2}$. By further restriction of $\mathcal{G}_{1}^{\prime}$, we will also make a specification on the size of the sumset of $\mathcal{G}_{\bar{x}_{1}, \bar{x}_{2}}$.

For $\left(\bar{x}_{1}, \bar{x}_{2}\right) \in \mathcal{G}_{1}^{\prime}$, let $K_{+}\left(\mathcal{G}_{\bar{x}_{1}, \bar{x}_{2}}\right)$ be the addition constant of $A_{1}\left(\bar{x}_{1}\right)$ and $A_{2}\left(\bar{x}_{2}\right)$ along the graph $\mathcal{G}_{\bar{x}_{1}, \bar{x}_{2}}$ as defined in Proposition 3. Let $\mathcal{H} \subset \mathcal{G}_{1}^{\prime}$, with

$$
|\mathcal{H}| \sim\left|\mathcal{G}_{1}^{\prime}\right|>\frac{\delta}{\left(\log \frac{K}{\delta}\right)^{3}} M_{1} M_{2} \text {. }
$$

Claim.

$$
\min _{\left(\bar{x}_{1}, \bar{x}_{2}\right) \in \mathcal{H}} K_{+}\left(\mathcal{G}_{\bar{x}_{1}, \bar{x}_{2}}\right)<L_{0} \equiv\left(\log \frac{K}{\delta}\right)^{\frac{9}{2}} \delta^{-\frac{9}{2}} K .
$$

Proof. Assume for all $\left(\bar{x}_{1}, \bar{x}_{2}\right) \in \mathcal{H}$ that $K_{+}\left(\mathcal{G}_{\bar{x}_{1}, \bar{x}_{2}}\right)>L_{0}$. Then

$$
\begin{aligned}
& K \sqrt{N_{1} N_{2}} \geq\left|A_{1} \underset{\mathcal{G}}{+} A_{2}\right|>\min _{\left(\bar{x}_{1}, \bar{x}_{2}\right) \in \mathcal{H}}\left\{\left|A_{1}\left(\bar{x}_{1}\right){\underset{\mathcal{G}}{\bar{x}_{1}, \bar{x}_{2}}}_{A_{2}} A_{2}\left(\bar{x}_{2}\right)\right|\right\}\left|\pi_{1}\left(A_{1}\right)+\pi_{\mathcal{H}}\left(A_{2}\right)\right| \\
& \geq L_{0} \sqrt{m_{1} m_{2}} \frac{|\mathcal{H}|}{\sqrt{M_{1} M_{2}}}>L_{0} \frac{c \delta}{\left(\log \frac{K}{\delta}\right)^{3}}\left(\overline{\bar{N}}_{1} \overline{\bar{N}}_{2}\right)^{1 / 2} \\
& >c \delta^{-1} \sqrt{N_{1} N_{2}} K
\end{aligned}
$$

which is a contradiction. (The last inequality is true by (3.23), (3.32) and (3.42).)

Hence, we may reduce $\mathcal{G}_{1}^{\prime}$ to $\mathcal{G}_{1}^{\prime \prime} \subset \mathcal{G}_{1}^{\prime}$, with $\left|\mathcal{G}_{1}^{\prime \prime}\right| \sim\left|\mathcal{G}_{1}^{\prime}\right|$ such that

$$
\left|A_{1}\left(\bar{x}_{1}\right)+_{\mathcal{G}_{\bar{x}_{1}, \bar{x}_{2}}} A_{2}\left(\bar{x}_{2}\right)\right|<L_{0} \sqrt{m_{1} m_{2}} \quad \text { for }\left(\bar{x}_{1}, \bar{x}_{2}\right) \in \mathcal{G}_{1}^{\prime \prime} .
$$

Therefore there is $\mathcal{G}_{1,0} \subset \mathcal{G}_{1}^{\prime \prime}$ and $L<L_{0}$ (see (3.42)),

$$
\left|\mathcal{G}_{1,0}\right|>\frac{\left|\mathcal{G}_{1}^{\prime \prime}\right|}{\log \frac{K}{\delta}}>\delta_{0} M_{1} M_{2},
$$


where, by (3.40)

$$
\delta_{0}>c \frac{\delta}{\delta_{1}\left(\log \frac{K}{\delta}\right)^{4}}
$$

and

$$
\left|A_{1}\left(\bar{x}_{1}\right)_{\mathcal{G}_{\bar{x}_{1}, \bar{x}_{2}}} A_{2}\left(\bar{x}_{2}\right)\right| \sim L \sqrt{m_{1} m_{2}} \quad \text { with } L<L_{0},
$$

for $\left(\bar{x}_{1}, \bar{x}_{2}\right) \in \mathcal{G}_{1,0}$.

Since

$$
\begin{aligned}
K \sqrt{N_{1} N_{2}} & \geq\left|\pi_{1}\left(A_{1}\right) \underset{\mathcal{G}_{1,0}}{+} \pi_{1}\left(A_{2}\right)\right|\left|A_{1}\left(\bar{x}_{1}\right){\underset{\mathcal{G}}{\bar{x}_{1}, \bar{x}_{2}}} A_{2}\left(\bar{x}_{2}\right)\right| \\
& \geq\left|\pi_{1}\left(A_{1}\right){\underset{\mathcal{G}}{\mathcal{G}_{1,0}}} \pi_{1}\left(A_{2}\right)\right| \cdot L \sqrt{m_{1} m_{2}} \\
& =K_{+}\left(\mathcal{G}_{1,0}\right) L \sqrt{\overline{\bar{N}}_{1} \overline{\bar{N}}_{2}},
\end{aligned}
$$

we have

$$
K_{+}\left(\mathcal{G}_{1,0}\right) \cdot L<\delta^{-\frac{5}{2}}\left(\log \frac{K}{\delta}\right)^{\frac{3}{2}} K<\delta^{-3}(\log K)^{2} K .
$$

In summary, $\mathcal{G}_{1,0} \subset \pi_{1}\left(A_{1}\right) \times \pi_{1}\left(A_{2}\right)$ satisfies (3.43), (3.44) and for $\left(\bar{x}_{1}, \bar{x}_{2}\right) \in \mathcal{G}_{1,0}$, the graph $\mathcal{G}_{\bar{x}_{1}, \bar{x}_{2}} \subset A_{1}\left(\bar{x}_{1}\right) \times A_{2}\left(\bar{x}_{2}\right)$ satisfies

$$
\begin{aligned}
& \left\{\left(\bar{x}_{1}, \bar{x}_{2}\right)\right\} \times \mathcal{G}_{\bar{x}_{1}, \bar{x}_{2}} \subset \mathcal{G}, \\
& \left|\mathcal{G}_{\bar{x}_{1}, \bar{x}_{2}}\right| \sim \delta_{1} m_{1} m_{2},
\end{aligned}
$$

where $\delta_{1}$ is as in (3.37). The addition constants $K_{+}\left(\mathcal{G}_{1,0}\right)$ and $L=K\left(\mathcal{G}_{\bar{x}_{1}, \bar{x}_{2}}\right)$ satisfy (3.45) and (3.46).

Denote

$$
\mathcal{G} \supset \tilde{\mathcal{G}}=\bigcup_{\left(\bar{x}_{1}, \bar{x}_{2}\right) \in \mathcal{G}_{1,0}}\left(\left\{\left(\bar{x}_{1}, \bar{x}_{2}\right)\right\} \times \mathcal{G}_{\bar{x}_{1}, \bar{x}_{2}}\right)
$$

which satisfies

$$
|\tilde{\mathcal{G}}|>c \frac{\delta}{\left(\log \frac{K}{\delta}\right)^{4}} \overline{\bar{N}}_{1} \overline{\bar{N}}_{2}
$$

where

$$
\overline{\bar{N}}_{1} \cdot \overline{\bar{N}}_{2}>\frac{\delta^{5}}{\left(\log \frac{K}{\delta}\right)^{3}} N_{1} N_{2} .
$$

Step 6. Moment inequalities.

Let $\tilde{\mathcal{G}}$ be the graph obtained in (3.48). We further reduce $\tilde{\mathcal{G}}$ to a graph $\mathcal{G}^{\prime}$ to fulfil condition (2.4).

Consider first the graph $\mathcal{G}_{1,0} \subset \pi_{1}\left(A_{1}\right) \times \pi_{1}\left(A_{2}\right) \subset \mathcal{R}_{1} \times \mathcal{R}_{1}$ and denote $K_{0}=$ $K_{+}\left(\mathcal{G}_{1,0}\right)$. By (3.43) and since $\phi, \psi$ are admissible, there is $\mathcal{G}_{1,0}^{\prime} \subset \mathcal{G}_{1,0}$ satisfying

$$
\left|\mathcal{G}_{1,0}^{\prime}\right|>\phi\left(M_{1} M_{2}, \delta_{0}, K_{0}\right)
$$

and

$$
\left\|\sum F_{\alpha}\left(\prod_{p \in \mathcal{P}_{1}} p^{\alpha_{p}} \theta\right)\right\|_{q} \leq \psi\left(M_{1} M_{2}, \delta_{0}, K_{0}\right)\left(\sum\left\|F_{\alpha}\right\|_{q}^{2}\right)^{1 / 2}
$$


whenever $\bar{x} \in \mathcal{R}_{1},\left(F_{\alpha}\right)_{\alpha \in \mathcal{G}_{1,0}^{\prime}(\bar{x})}$ trigonometric polynomials, satisfying

$$
(n, p)=1 \text {, for any } n \in \operatorname{supp} \widehat{F}_{\alpha} \text { and for any } p \in \mathcal{P}_{1} \text {. }
$$

Next, fix $\left(\bar{x}_{1}, \bar{x}_{2}\right) \in \mathcal{G}_{1,0}^{\prime}$ and consider the graph $\mathcal{G}_{\bar{x}_{1}, \bar{x}_{2}} \subset A_{1}\left(\bar{x}_{1}\right) \times A_{2}\left(\bar{x}_{2}\right) \subset \mathcal{R}_{2} \times \mathcal{R}_{2}$ satisfying (3.47) and (3.45).

Thus there is a subgraph $\mathcal{G}_{\bar{x}_{1}, \bar{x}_{2}}^{\prime} \subset \mathcal{G}_{\bar{x}_{1}, \bar{x}_{2}}$ s.t.

$$
\left|\mathcal{G}_{\bar{x}_{1}, \bar{x}_{2}}^{\prime}\right|>\phi\left(m_{1} m_{2}, \delta_{1}, L\right)
$$

and

$$
\left\|\sum G_{\alpha}\left(\prod_{p \in \mathcal{P}_{2}} p^{\alpha_{p}} \theta\right)\right\|_{q} \leq \psi\left(m_{1} m_{2}, \delta_{1}, L\right)\left(\sum\left\|G_{\alpha}\right\|_{q}^{2}\right)^{1 / 2}
$$

whenever $\bar{y} \in \mathcal{R}_{2}$ and $\left(G_{\alpha}\right)_{\alpha \in \mathcal{G}_{\bar{x}_{1}, \bar{x}_{2}}^{\prime}(\bar{y})}$ are trigonometric polynomials satisfying

$$
(n, p)=1 \text {, for any } n \in \operatorname{supp} \widehat{G}_{\alpha} \text { and for any } p \in \mathcal{P}_{2} \text {. }
$$

Consider then the subgraph $\mathcal{G}^{\prime} \subset \tilde{\mathcal{G}} \subset \mathcal{G}$,

$$
\mathcal{G}^{\prime}=\bigcup_{\left(\bar{x}_{1}, \bar{x}_{2}\right) \in \mathcal{G}_{1,0}^{\prime}}\left(\left\{\left(\bar{x}_{1}, \bar{x}_{2}\right)\right\} \times \mathcal{G}_{\bar{x}_{1}, \bar{x}_{2}}^{\prime}\right),
$$

which satisfies by (3.51) and (3.53)

$$
\left|\mathcal{G}^{\prime}\right|>\phi\left(M_{1} M_{2}, \delta_{0}, K_{0}\right) \cdot \phi\left(m_{1} m_{2}, \delta_{1}, L\right) .
$$

Next, we check the moment inequality.

Fix thus $x=(\bar{x}, \bar{y}) \in \mathcal{R}_{1} \times \mathcal{R}_{2}$ and consider trigonometric polynomials $\left(G_{\alpha}\right)_{\alpha \in \mathcal{G}^{\prime}(x)}$, where $\mathcal{G}^{\prime}(x)=\left\{\alpha \mid(x, \alpha) \in \mathcal{G}^{\prime} \subset A \times A\right\}$, such that

$$
(n, p)=1 \text {, for any } n \in \operatorname{supp} \widehat{G}_{\alpha} \text { and for any } p \in \mathcal{P}_{0}=\mathcal{P}_{1} \cup \mathcal{P}_{2} \text {. }
$$

We need to estimate $\left\|\sum_{\alpha} G_{\alpha}\left(\prod_{p \in \mathcal{P}_{0}} p^{\alpha_{p}} \theta\right)\right\|_{q}$ using (3.52) and (3.54). First, by $(3.55)$,

$$
\mathcal{G}^{\prime}(x)=\bigcup_{\beta \in \mathcal{G}_{1,0}^{\prime}(\bar{x})}\left(\{\beta\} \times \mathcal{G}_{\bar{x}, \beta}^{\prime}(\bar{y})\right) \subset \mathcal{R}_{0} .
$$

Denote for $\beta \in \mathcal{G}_{1,0}^{\prime}(\bar{x})$

$$
F_{\beta}(\theta)=\sum_{\pi_{1}(\alpha)=\beta} G_{\alpha}\left(\prod_{p \in \mathcal{P}_{2}} p^{\alpha_{p}} \theta\right)
$$

which clearly satisfy $(*)$.

Hence, applying (3.54) and (3.52) consecutively,

$$
\begin{aligned}
\left\|\sum_{\alpha} G_{\alpha}\left(\prod_{p \in \mathcal{P}_{0}} p^{\alpha_{p}} \theta\right)\right\|_{q} & =\left\|\sum_{\beta \in \mathcal{G}_{1,0}^{\prime}(\bar{x})} F_{\beta}\left(\prod_{p \in \mathcal{P}_{1}} p^{\beta_{p}} \theta\right)\right\|_{q} \\
& \leq \psi\left(M_{1} M_{2}, \delta_{0}, K_{0}\right)\left(\sum\left\|F_{\beta}\right\|_{q}^{2}\right)^{1 / 2} \\
& \leq \psi\left(M_{1} M_{2}, \delta_{0}, K_{0}\right) \cdot \psi\left(m_{1} m_{2}, \delta_{1}, L\right)\left(\sum_{\alpha}\left\|G_{\alpha}\right\|_{q}^{2}\right)^{1 / 2} .
\end{aligned}
$$


Returning to the statement in Lemma 3.1 and inequalities (3.56) and (3.57), we get in both (3.2) and (3.3)

$$
\left\{\begin{array}{l}
N^{\prime}=M_{1} M_{2}, \quad N^{\prime \prime}=m_{1} m_{2}, \\
\delta^{\prime}=\delta_{0}, \quad \delta^{\prime \prime}=\delta_{1} \\
K^{\prime}=K_{0}, \quad K^{\prime \prime}=L
\end{array}\right.
$$

Condition (3.4) follows from (3.50) (which is clearly much stronger). Restating (3.44) and (3.46) gives

$$
\begin{aligned}
\delta_{0} \delta_{1} & >\frac{\delta}{\left(\log \frac{K}{\delta}\right)^{4}}, \\
K_{0} L & <\delta^{-3}(\log K)^{2} K .
\end{aligned}
$$

It remains to consider condition (3.5).

By (3.24) and (3.34)

$$
M_{1} M_{2}<C \delta^{-15} K^{7} N^{1 / 2}
$$

but we do not necessarily have the desired bound on $m_{1} m_{2}$. To achieve this, we will redefine $\mathcal{G}_{\bar{x}_{1}, \bar{x}_{2}}^{\prime}$ by performing one more step in the construction.

Step 7. Recalling Step 2, decompose $\mathcal{P}_{2}=\left\{p_{t^{\prime}+1}, \ldots, p_{t}\right\}$ further as

$$
\mathcal{P}_{2}=\left\{p_{t^{\prime}+1}\right\} \cup \mathcal{P}_{3}, \text { where } \mathcal{P}_{3}=\left\{p_{t^{\prime}+2}, \ldots, p_{t}\right\} .
$$

For fixed $\left(\bar{x}_{1}, \bar{x}_{2}\right) \in \mathcal{G}_{1,0}^{\prime}$, consider the graph $\mathcal{K}=\mathcal{G}_{\bar{x}_{1}, \bar{x}_{2}} \subset A_{1}\left(\bar{x}_{1}\right) \times A_{2}\left(\bar{x}_{2}\right) \subset$ $\mathcal{R}_{2} \times \mathcal{R}_{2}$ satisfying by (3.45) and (3.47)

$$
\begin{aligned}
& \left|A_{1}\left(\bar{x}_{1}\right)\right| \sim m_{1},\left|A_{2}\left(\bar{x}_{2}\right)\right| \sim m_{2}, \\
& \left|\mathcal{G}_{\bar{x}_{1}, \bar{x}_{2} \mid}\right| \sim \delta_{1} m_{1} m_{2}, \\
& K_{+}\left(\mathcal{G}_{\bar{x}_{1}, \bar{x}_{2}}\right) \sim L .
\end{aligned}
$$

Then repeat Steps $1-5$ from previous construction to the graph $\mathcal{K}$ with respect to the decomposition $\mathcal{P}_{2}=\left\{p_{t^{\prime}+1}\right\} \cup \mathcal{P}_{3}$. Thus $\mathcal{K}$ gets replaced by

$$
\tilde{\mathcal{K}}=\bigcup_{\left(z_{1}, z_{2}\right) \in \mathcal{K}_{1,0}}\left\{\left(z_{1}, z_{2}\right)\right\} \times \mathcal{K}_{z_{1}, z_{2}}
$$

where

$$
\mathcal{K}_{1,0} \subset\left(\mathbb{Z}_{\geq 0}\right)^{2} .
$$

Thus $\tilde{\mathcal{K}} \subset \overline{\overline{A_{1}\left(\bar{x}_{1}\right)}} \times \subset \overline{\overline{A_{2}\left(\bar{x}_{2}\right)}} \subset A_{1}\left(\bar{x}_{1}\right) \times A_{2}\left(\bar{x}_{2}\right)$ (cf. (3.23), (3.32))

$$
\begin{aligned}
& \mathcal{K}_{z_{1}, z_{2}} \subset \overline{\overline{A_{1}\left(\bar{x}_{1}\right)}}\left(z_{1}\right) \times \overline{\overline{A_{2}\left(\bar{x}_{2}\right)}}\left(z_{2}\right), \\
& m_{i} \geq\left|\overline{\overline{A_{i}\left(\bar{x}_{i}\right)}}\right| \equiv \overline{\bar{m}}_{i}>\frac{\delta_{1}^{3}}{\left(\log \frac{L}{\delta_{1}}\right)^{2}} m_{i}, \\
& \left|\overline{\overline{A_{i}\left(\overline{\left.x_{i}\right)}\right.}}\left(z_{i}\right)\right| \sim \ell_{i} \leq\left|A_{i}\left(\bar{x}_{i}, z_{i}\right)\right|<\left(N_{1} N_{2}\right)^{1 / 4} .
\end{aligned}
$$

The last inequality is true by (3.12).

$$
\begin{aligned}
\left|\mathcal{K}_{z_{1}, z_{2}}\right| & \sim \delta_{3} \ell_{1} \ell_{2}, \\
\left|\mathcal{K}_{1,0}\right| & >\frac{\delta_{1}}{\delta_{3}\left(\log \frac{L}{\delta_{1}}\right)^{4}} \frac{\overline{\bar{m}}_{1} \overline{\bar{m}}_{2}}{\ell_{1} \ell_{2}}
\end{aligned}
$$


(cf. (3.43), (3.44))

$$
K\left(\mathcal{K}_{z_{1}, z_{2}}\right)<K\left(\mathcal{K}_{1,0}\right) \cdot K\left(\mathcal{K}_{z_{1}, z_{2}}\right)<\delta_{1}^{-3}(\log L)^{2} L
$$

(cf. (3.46)).

(We point out here that $\ell_{i}, \overline{\bar{m}}_{i}, \delta_{3}>\frac{\delta_{1}}{\left(\log \frac{L}{\delta_{1}}\right)^{2}}$ do depend on the basepoint $\left(\bar{x}_{1}, \bar{x}_{2}\right)$ $\in \mathcal{R}_{1} \times \mathcal{R}_{1}$.) Starting from (3.60), we carry out Step 6. However, since $\mathcal{K}_{1,0} \subset\left(\mathbb{Z}_{\geq 0}\right)^{2}$ (only the prime $p_{t^{\prime}+1}$ is involved), we may take $\mathcal{K}_{1,0}^{\prime}=\mathcal{K}_{1,0}$ and replace in (3.52) the factor $\psi\left(\right.$ ) by $C q$ (apply Proposition 1 with $k=1$ ). For each $\left(z_{1}, z_{2}\right) \in \mathcal{K}_{1,0}$, consider again a subgraph $\mathcal{K}_{z_{1}, z_{2}}^{\prime} \subset \mathcal{K}_{z_{1}, z_{2}}$ satisfying

$$
\left|\mathcal{K}_{z_{1}, z_{2}}^{\prime}\right|>\phi\left(\ell_{1} \ell_{2}, \delta_{3}, K\left(\mathcal{K}_{z_{1}, z_{2}}\right)\right)
$$

and

$$
\left\|\sum G_{\alpha}\left(\prod_{p \in \mathcal{P}_{3}} p^{\alpha_{p}} \theta\right)\right\|_{q}=\psi\left(\ell_{1} \ell_{2}, \delta_{3}, K\left(\mathcal{K}_{z_{1}, z_{2}}\right)\right)\left(\sum\left\|G_{\alpha}\right\|_{q}^{2}\right)^{1 / 2}
$$

whenever $\left(G_{\alpha}\right)_{\alpha \in \mathcal{K}_{z_{1}, z_{2}}^{\prime}(\bar{y})}$ are trigonometric polynomials satisfying

$$
(n, p)=1, \text { for any } n \in \operatorname{supp} \widehat{G}_{\alpha} \text { and for any } p \in \mathcal{P}_{3} .
$$

Then redefine $\mathcal{G}_{\bar{x}_{1}, \bar{x}_{2}}^{\prime} \subset \mathcal{G}_{\bar{x}_{1}, \bar{x}_{2}}$ as

$$
\mathcal{G}_{\bar{x}_{1}, \bar{x}_{2}}^{\prime}=\bigcup_{\left(z_{1}, z_{2}\right) \in \mathcal{K}_{1,0}}\left(\left\{\left(z_{1}, z_{2}\right)\right\} \times \mathcal{K}_{z_{1}, z_{2}}^{\prime}\right)
$$

and again take

$$
\mathcal{G}^{\prime}=\bigcup_{\left(\bar{x}_{1}, \bar{x}_{2}\right) \in \mathcal{G}_{1,0}^{\prime}}\left(\left\{\left(\bar{x}_{1}, \bar{x}_{2}\right)\right\} \times \mathcal{G}_{\bar{x}_{1}, \bar{x}_{2}}^{\prime}\right) .
$$

From the preceding, since $\ell_{1} \ell_{2} \leq \min \left\{m_{1} m_{2}, N^{1 / 2}\right\}$ in (3.61) and (3.62), the factor in the moment bound (3.57) now becomes

$$
C q \psi\left(M_{1} M_{2}, \delta_{0}, K_{0}\right) \cdot \psi\left(\min \left\{m_{1} m_{2}, N^{1 / 2}\right\}, \frac{\delta_{1}}{\left(\log \frac{L}{\delta_{1}}\right)^{2}}, \delta_{1}^{-3}(\log L)^{2} L\right)
$$

Thus by (3.59), in (3.3), $N^{\prime}=M_{1} M_{2}, N^{\prime \prime}=\min \left\{m_{1} m_{2}, N^{1 / 2}\right\}$ satisfy (3.5) (and may be increased to also satisfy the lower bound in (3.4)).

Also, by (3.37) and (3.42) (which imply $\log \frac{L}{\delta_{1}}<c \log \frac{K}{\delta}$ ), together with (3.58), we have

which is condition (3.6).

$$
\delta_{0} \cdot \frac{\delta_{1}}{\left(\log \frac{L}{\delta_{1}}\right)^{2}}>c \frac{\delta}{\left(\log \frac{K}{\delta}\right)^{6}},
$$

Taking $K^{\prime}=K_{0}, K^{\prime \prime}=\delta_{1}^{-3}(\log L)^{2} L,(3.58)$ and (3.37) imply

$$
\begin{aligned}
K^{\prime} \cdot K^{\prime \prime} & <\delta^{-6}(\log K)^{2}\left(\log \frac{K}{\delta}\right)^{6}(\log L)^{2} K \\
& <\delta^{-14}(\log K)^{2}\left(\delta \log \frac{K}{\delta}\right)^{6}(\delta \log L)^{2} K \\
& <\delta^{-14}(\log K)^{10} K
\end{aligned}
$$

and hence (3.7) holds. 
From (3.64)-(3.66), (3.68) and (3.61),

$$
\begin{aligned}
\left|\mathcal{G}_{\bar{x}_{1}, \bar{x}_{2}}^{\prime}\right| & >\left\{1+\frac{\delta_{1}}{\delta_{3}\left(\log \frac{K}{\delta}\right)^{4}} \frac{\overline{\bar{m}}_{1} \overline{\bar{m}}_{2}}{\ell_{1} \ell_{2}}\right\} \cdot \phi\left(\ell_{1} \ell_{2}, \delta_{3}, \delta_{1}^{-3}(\log L)^{2} L\right) \\
& >\left\{1+\frac{\delta_{1}^{7}}{\left(\log \frac{K}{\delta}\right)^{8}} \frac{m_{1} m_{2}}{\ell_{1} \ell_{2}}\right\} \cdot \phi\left(\ell_{1} \ell_{2}, \frac{\delta_{1}}{\left(\log \frac{K}{\delta}\right)^{2}}, \delta_{1}^{-3}(\log L)^{2} L\right) .
\end{aligned}
$$

Define

$$
N^{\prime \prime}=\min \left\{N^{1 / 2}, 1+\frac{\delta_{1}^{7}}{\left(\log \frac{K}{\delta}\right)^{8}} m_{1} m_{2}\right\} .
$$

Using property (3.1) of the function $\phi$, we verify that

$$
\left|\mathcal{G}_{\bar{x}_{1}, \bar{x}_{2}}^{\prime}\right|>\left(1+\frac{\delta_{1}^{7} m_{1} m_{2}}{\left(\log \frac{K}{\delta}\right)^{8}}\right) \frac{1}{N^{\prime \prime}} \cdot \phi\left(N^{\prime \prime}, \frac{\delta_{1}}{\left(\log \frac{K}{\delta}\right)^{2}}, \delta_{1}^{-3}(\log L)^{2} L\right) .
$$

Hence, again by (3.1) and (3.51)

$$
\begin{aligned}
\left|\mathcal{G}^{\prime}\right|> & \left(1+\frac{\delta_{1}^{7} m_{1} m_{2}}{\left(\log \frac{K}{\delta}\right)^{8}}\right) \frac{1}{N^{\prime \prime}} \cdot \phi\left(M_{1} M_{2}, \delta_{0}, K_{0}\right) \\
& \cdot \phi\left(N^{\prime \prime}, \frac{\delta_{1}}{\left(\log \frac{K}{\delta}\right)^{2}}, \delta_{1}^{-3}(\log L)^{2} L\right) \\
> & \phi\left(N^{\prime}, \delta_{0}, K_{0}\right) \cdot \phi\left(N^{\prime \prime}, \frac{\delta_{1}}{\left(\log \frac{K}{\delta}\right)^{2}}, \delta_{1}^{-3}(\log L)^{2} L\right)
\end{aligned}
$$

denoting

$$
N^{\prime}=\left(1+\frac{\delta_{1}^{7} m_{1} m_{2}}{\left(\log \frac{K}{\delta}\right)^{8}}\right) \frac{M_{1} M_{2}}{N^{\prime \prime}} .
$$

Thus (3.37) and (3.50) imply

$$
N>N^{\prime} N^{\prime \prime}>\frac{\delta_{1}^{7}}{\left(\log \frac{K}{\delta}\right)^{8}} \cdot \overline{\bar{N}}_{1} \overline{\bar{N}}_{2}>\delta^{12}\left(\log \frac{K}{\delta}\right)^{-25} .
$$

This implies (3.4).

By (3.70), (3.72) and (3.59)

$$
N^{\prime \prime} \leq N^{1 / 2}, \quad N^{\prime}<M_{1} M_{2}+\frac{\overline{\bar{N}}_{1} \overline{\bar{N}}_{2}}{N^{1 / 2}}<C \delta^{-15} K^{7} N^{1 / 2},
$$

which implies (3.5).

This proves Lemma 3.1 .

\section{Proof of Proposition 3, Part II}

Recalling (2.13) and (2.14), we start from the pair of admissible functions

$$
\begin{aligned}
\phi(N, \delta, K) & =\left(\frac{\delta}{K}\right)^{C} N, \\
\psi(N, \delta, k) & =\min \left(q^{\left(\frac{K}{\delta}\right)^{C}}, N^{1 / 2}\right)
\end{aligned}
$$


( $C=$ some constant). The $N^{\frac{1}{2}}$-bound in (4.2) is obtained from the obvious estimate

$$
\left\|\sum_{\alpha} F_{\alpha}\left(\prod_{p \in \mathcal{P}_{0}} p^{\alpha_{p}} \theta\right)\right\|_{q} \leq \sum_{\alpha}\left\|F_{\alpha}\right\|_{q} \leq N^{1 / 2}\left(\sum\left\|F_{\alpha}\right\|_{q}^{2}\right)^{1 / 2}
$$

(since $\alpha$ ranges in a set of size at most $N$ ).

Starting from (4.1), (4.2), we produce here a new pair of admissible functions by applying Lemma 3.1. The next statement does not yet imply Proposition 2 but already displays a much better behavior of $\psi$ in $K$.

Lemma 4.1. Take

$$
\tilde{\phi}(N, \delta, K)=\left(\frac{\delta}{K}\right)^{C \log \log \frac{K}{\delta}} \cdot N
$$

and

$$
\tilde{\psi}(N, \delta, K)=q^{\left(\log \frac{K}{\delta}\right)^{C / \gamma}} \cdot N^{\gamma}
$$

with $C$ an appropriate constant and $0<\gamma<1$ arbitrary.

Then $\tilde{\phi}, \tilde{\psi}$ are admissible.

Proof. We will make an iterated application of Lemma 3.1.

Fix $N, \delta, K$ and choose an integer $t$ of the form $2^{\ell}$ (to be specified). Starting from $\phi_{0}=\phi, \psi_{0}=\psi$, define recursively for $\ell^{\prime}=0,1, \ldots, \ell-1$,

$$
\begin{aligned}
& \phi_{\ell^{\prime}+1}(N, \delta, K)=\min \phi_{\ell^{\prime}}\left(N^{\prime}, \delta^{\prime}, K^{\prime}\right) \cdot \phi_{\ell^{\prime}}\left(N^{\prime \prime}, \delta^{\prime \prime}, K^{\prime \prime}\right), \\
& \psi_{\ell^{\prime}+1}(N, \delta, K)=C q \max \psi_{\ell^{\prime}}\left(N^{\prime}, \delta^{\prime}, K^{\prime}\right) \cdot \psi_{\ell^{\prime}}\left(N^{\prime \prime}, \delta^{\prime \prime}, K^{\prime \prime}\right),
\end{aligned}
$$

where in (4.6), (4.7) the parameters $N^{\prime}, N^{\prime \prime}, \delta^{\prime}, \delta^{\prime \prime}, K^{\prime}, K^{\prime \prime}$ satisfy (3.4)-(3.7).

We evaluate $\tilde{\phi}=\phi_{\ell}, \tilde{\psi}=\psi_{\ell}$.

Iterating (4.6), we clearly obtain

$$
\tilde{\phi}(N, \delta, K)=\prod_{\nu \in\{0,1\}^{\ell}} \phi\left(N_{\nu}, \delta_{\nu}, K_{\nu}\right)
$$

where

$$
\left(N_{\nu}\right)_{\nu \in} \cup\{0,1\}^{\ell^{\prime}}, \quad\left(\delta_{\nu}\right)_{\nu \in \in_{\ell^{\prime} \leq \ell}\{0,1\}^{\ell^{\prime}}}, \quad\left(K_{\nu}\right)_{\nu \in} \cup\{0,1\}^{\ell^{\prime}}
$$

satisfy by (3.4)-(3.7) the constraints

$$
\begin{aligned}
N_{\phi} & =N, \quad \delta_{\phi}=\delta, \quad K_{\phi}=K \\
N_{\nu} \geq & N_{\nu, 0} \cdot N_{\nu, 1} \geq N_{\nu}\left(\frac{\delta_{\nu}}{\log K_{\nu}}\right)^{40}, \\
N_{\nu, 0}+N_{\nu, 1}< & \left(\frac{K_{\nu}}{\delta_{\nu}}\right)^{15} N_{\nu}^{1 / 2} \\
\delta_{\nu, 0} \cdot \delta_{\nu, 1} & >\left(\log \frac{K_{\nu}}{\delta_{\nu}}\right)^{-6} \delta_{\nu}, \\
K_{\nu, 0} \cdot K_{\nu, 1} & \leq \delta_{\nu}^{-14}\left(\log K_{\nu}\right)^{10} K_{\nu} .
\end{aligned}
$$

From (4.12), (4.13)

$$
\log \frac{K_{\nu, 0}}{\delta_{\nu, 0}}+\log \frac{K_{\nu, 1}}{\delta_{\nu, 1}}<21 \log \frac{K_{\nu}}{\delta_{\nu}}
$$


and iteration implies

$$
\max _{\nu \in\{0,1\}^{\ell^{\prime}}} \log \frac{K_{\nu}}{\delta_{\nu}} \leq \sum_{\nu \in\{0,1\}^{\ell^{\prime}}} \log \frac{K_{\nu}}{\delta_{\nu}}<21^{\ell^{\prime}} \log \frac{K}{\delta} .
$$

Iteration of (4.12) gives

$$
\begin{aligned}
\prod_{\nu \in\{0,1\}^{\ell^{\prime}}} \delta_{\nu} & >\prod_{\nu \in\{0,1\}^{\ell^{\prime}-1}}\left(\log \frac{K_{\nu}}{\delta_{\nu}}\right)^{-6} \prod_{\nu \in\{0,1\}^{\ell^{\prime}-1}} \delta_{\nu} \\
& \stackrel{(4.14)}{>} 21^{-3 \ell^{\prime} 2^{\ell^{\prime}}}\left(\log \frac{K}{\delta}\right)^{-3 \cdot 2^{\ell^{\prime}}} \prod_{\nu \in\{0,1\}^{\ell^{\prime}-1}} \delta_{\nu} \\
& >21^{-3\left(\ell^{\prime} 2^{\ell^{\prime}}+\left(\ell^{\prime}-1\right) 2^{\ell^{\prime}-1}+\cdots\right)}\left(\log \frac{K}{\delta}\right)^{-3\left(2^{\ell^{\prime}}+2^{\ell^{\prime}-1}+\cdots\right)} \delta \\
& >21^{-6 \ell^{\prime} 2^{\ell^{\prime}}}\left(\log \frac{K}{\delta}\right)^{-6 \cdot 2^{\ell^{\ell^{\prime}}}} \delta .
\end{aligned}
$$

Similarly, iterate (4.13). Thus $(4.14)$ and $(4,15)$ give

$$
\begin{aligned}
\prod_{\nu \in\{0,1\}^{\ell^{\prime}}} K_{\nu} & \leq \prod_{\nu \in\{0,1\}^{\ell^{\prime}-1}} \delta_{\nu}^{-14}\left(\log K_{\nu}\right)^{10} \cdot \prod_{\nu \in\{0,1\}^{\ell^{\prime}-1}} K_{\nu} \\
& <\left(21^{-3 \ell^{\prime} 2^{\ell^{\prime}}}\left(\log \frac{K}{\delta}\right)^{-3 \cdot 2^{\ell^{\prime}}} \delta\right)^{-14}\left(21^{\ell^{\prime}} \log \frac{K}{\delta}\right)^{5 \cdot 2^{\ell^{\prime}}}\left(\prod_{\nu \in\{0,1\}^{\ell^{\prime}-1}} K_{\nu}\right) \\
(4.16) & <21^{94 \ell^{\prime} 2^{\ell^{\prime}}}\left(\log \frac{K}{\delta}\right)^{94 \cdot 2^{\ell^{\prime}}} \delta^{-14 \ell^{\prime}} K .
\end{aligned}
$$

By (4.10), (4.14) and (4.15),

$$
\begin{aligned}
\prod_{\nu \in\{0,1\}^{\ell^{\prime}}} N_{\nu} & >\prod_{\nu \in\{0,1\}^{\ell^{\prime}-1}}\left(\frac{\delta_{\nu}}{\log K_{\nu}}\right)^{40} \cdot \prod_{\nu \in\{0,1\}^{\ell^{\prime}-1}} N_{\nu} \\
& >21^{-140 \ell^{\prime} 2^{\ell^{\prime}}} \delta^{40}\left(21^{\ell^{\prime}} \log \frac{K}{\delta}\right)^{-140 \cdot 2^{\ell^{\prime}}} \prod_{\nu \in\{0,1\}^{\ell^{\prime}-1}} N_{\nu} \\
& >21^{-280 \ell^{\prime} 2^{\ell^{\prime}}}\left(\log \frac{K}{\delta}\right)^{-2802^{\ell^{\prime}}} \delta^{40 \ell^{\prime}} N .
\end{aligned}
$$

Substitution of (4.1) in (4.8) gives by (4.15), (4.16), (4.17)

$$
\begin{aligned}
\tilde{\phi}(N, \delta, K) & \geq \prod_{\nu \in\{0,1\}^{\ell}}\left(\frac{\delta_{\nu}}{K_{\nu}}\right)^{C} N_{\nu} \\
& >e^{-C^{\prime} \ell 2^{\ell}}\left(\log \frac{K}{\delta}\right)^{-C^{\prime} 2^{\ell}} \delta^{C^{\prime} \ell} K^{-C} N \\
& >t^{-C^{\prime} t}\left(\log \frac{K}{\delta}\right)^{-C^{\prime} t}\left(\delta^{C^{\prime} \log t}\right) K^{-C} N .
\end{aligned}
$$


Take

$$
2^{\ell}=t \sim \log \frac{K}{\delta}
$$

Substitution of (4.19) in (4.18) gives (4.4).

Similarly, we will iterate (4.7) with (possibly different) parameters $\left(N_{\nu}\right),\left(\delta_{\nu}\right)$, $\left(K_{\nu}\right)$ still satisfying $(4.9)-(4.17)$.

By (4.2)

$$
\tilde{\psi}(N, \delta, K)=(C q)^{t} \prod_{\nu \in\{0,1\}^{\ell}} \min \left(q^{\left(\frac{K_{\nu}}{\delta_{\nu}}\right)^{C}}, N_{\nu}^{1 / 2}\right)
$$

From $(4.12)\left(\right.$ which implies that $\left.\delta_{\nu, 0}, \delta_{\nu, 1}>\left(\log \frac{K_{\nu}}{\delta_{\nu}}\right)^{-6} \delta_{\nu}\right)$ and $(4.14)$

$$
\delta_{\nu}>21^{-6 \ell^{2}}\left(\log \frac{K}{\delta}\right)^{-6 \ell} \delta
$$

and from (4.13) (which implies that $\left.K_{\nu, 0}, K_{\nu, 1} \leq \delta_{\nu}^{-14}\left(\log K_{\nu}\right){ }^{10} K_{\nu}\right),(4.14)$, and $(4.21)$

$$
K_{\nu}<21^{85 \ell^{3}}\left(\log \frac{K}{\delta}\right)^{85 \ell^{2}} \delta^{-14 \ell} K
$$

Hence from (4.11), (4.21), (4.22)

$$
\begin{aligned}
& N_{\nu, 0}+N_{\nu, 1}<21^{600 \ell^{3}}\left(\log \frac{K}{\delta}\right)^{600 \ell^{2}} \delta^{-600 \ell} K^{15} N_{\nu}^{1 / 2} \\
& N_{\nu}<\left(21^{600 \ell^{3}}\left(\log \frac{K}{\delta}\right)^{600 \ell^{2}} \delta^{-600 \ell} K^{15}\right)^{\left(1+\frac{1}{2}+\frac{1}{4}+\cdots\right)} N^{\frac{1}{2^{\ell}}} \\
& <10^{10^{3} \ell^{3}}\left(\log \frac{K}{\delta}\right)^{10^{4} \ell^{2}} \delta^{-10^{4} \ell} K^{30} N^{\frac{1}{t}} \quad \text { for } \nu \in\{0,1\}^{\ell}
\end{aligned}
$$

To bound (4.20), let $A$ be a number to be specified and partition

$$
\{0,1\}^{\ell}=I \cup J \quad \text { with } I=\left\{\nu \in\{0,1\}^{\ell} \mid \frac{K_{\nu}}{\delta_{\nu}} \leq A\right\} .
$$

It follows then from (4.20) and (4.23) that

$$
\tilde{\psi}(N, \delta, K)<q^{t A^{C}}\left[C^{\ell^{3}}\left(\log \frac{K}{\delta}\right)^{10^{4} \ell^{2}} \delta^{-10^{4} \ell} K^{30} N^{\frac{1}{t}}\right]^{|J|} .
$$

Now, we will estimate $|J|$.

From (4.15), (4.16)

$$
A^{|J|} \leq \prod_{\nu \in\{0,1\}^{\ell}} \frac{K_{\nu}}{\delta_{\nu}}<21^{100 \ell t} \cdot\left(\log \frac{K}{\delta}\right)^{100 t} \delta^{-15 \ell} K .
$$

Fixing $0<\gamma<1$, take

$$
\log A \sim \gamma^{-1} \log t
$$


It follows then from (4.25) that

$$
|J|<\frac{10^{3} t \cdot \log t}{\log A}<\gamma t
$$

Hence clearly from (4.24)

$$
\tilde{\psi}(N, \delta, K)<q^{t A^{C}} e^{t^{2} \log t} N^{\gamma}<q^{\left(\log \frac{K}{\delta}\right)^{C / \gamma}} \cdot N^{\gamma},
$$

which is (4.5).

This proves Lemma 4.1 .

\section{Proof of Proposition 3, Part III}

We will first use Lemma 3.1 and Lemma 4.1 to show

Lemma 5.1. Assume again the moment $q$ fixed. Given $0<\tau, \gamma<\frac{1}{2}$, for $i=$ $1,2,3$, there are positive constants $A_{i}=A_{i}(\tau, \gamma), B_{i}=B_{i}(\tau, \gamma)$ such that taking $N$ sufficiently large

$$
\begin{aligned}
& \phi(N, \delta, K)=K^{-A_{1}} \delta^{A_{2} \log \log N} e^{A_{3}(\log \log N)^{2}} N^{1-\tau}, \\
& \psi(N, \delta, K)=K^{B_{1}} \delta^{-B_{2} \log \log N} e^{-B_{3}(\log \log N)^{2}} N^{\gamma}
\end{aligned}
$$

is a pair of admissible functions.

Proof. We will proceed in two steps.

First, some notation.

Notation. We use " $\ell \ell$ " to denote "log log".

It follows from Lemma 4.1 (by taking $\frac{\gamma}{4}$ and assuming $\frac{K}{\delta}<N$ ) that

$$
\begin{aligned}
& \phi(N, \delta, K)=\left(\frac{\delta}{K}\right)^{C_{0} \ell \ell N} N, \\
& \psi(N, \delta, K)=\min \left\{\exp \left[\log q \cdot\left(\log \frac{K}{\delta}\right)^{\frac{4 C_{0}}{\gamma}}\right] \cdot N^{\frac{\gamma}{4}}, N^{1 / 2}\right\}
\end{aligned}
$$

are admissible.

First, fix a large integer $\bar{N}$ (depending on $\tau, \gamma$ ) and define

$$
A_{3} \sim A_{2} \sim A_{1}=C_{0} \ell \ell \bar{N}
$$

( $A_{2}, A_{3}$ will be specified later).

Thus the expression in the right-hand side of (5.1) is at most

$$
\left(\frac{\delta}{K}\right)^{C_{0} \ell \ell \bar{N}} e^{C(\ell \ell \bar{N})^{3}} N^{1-\tau}<\left(\frac{\delta}{K}\right)^{C_{0} \ell \ell N} N=\phi(N, \delta, K),
$$

if $\bar{N}$ is large enough and $N \leq \bar{N}, \log N \sim \log \bar{N}$.

Taking $N \leq \bar{N}$, then $(5.4)<N^{\frac{\gamma}{2}}<(5.2)$, provided

$$
\log q \cdot\left(\log \frac{K}{\delta}\right)^{\frac{4 C_{0}}{\gamma}}<\frac{\gamma}{4} \log N
$$

and

$$
e^{B_{3}(\ell \ell N)^{2}}<N^{\frac{\gamma}{2}} .
$$

If (5.6) does not hold, then for some $c>0$,

$$
\frac{K}{\delta}>e^{(\log N)^{c \gamma}}
$$


Thus if we take

$$
B_{3} \sim B_{2} \sim B_{1}=(\log \bar{N})^{1-c \gamma}
$$

(also $B_{2}, B_{3}$ to be specified later), (5.8) implies that (5.2) >N if $N \leq \bar{N}$ and (5.7) holds. From (5.9) and this choice of $B_{3}$, clearly (5.7) holds for $N \leq \bar{N}$, $\log N \sim \log \bar{N}$. Thus with the preceding choice of $A_{1}, A_{2}, A_{3}, B_{1}, B_{2}, B_{3}$, the pair of functions (5.1), (5.2) is admissible in the range $N \leq \bar{N}, \log N \sim \log \bar{N}$.

Next we use Lemma 3.1 to establish that (5.1), (5.2) are also admissible in the range $N>\bar{N}$. We will proceed by induction on the size of $N$. Obviously $(5.2)>N^{1 / 2}$ if $\left(\frac{K}{\delta}\right)^{B_{1}}>N^{1 / 2}$. Hence we may assume

$$
\frac{K}{\delta}<N^{10^{-6}}
$$

We want to reduce $N$ by Lemma 3.1. Thus we estimate

$$
\phi\left(N^{\prime}, \delta^{\prime}, K^{\prime}\right) \cdot \phi\left(N^{\prime \prime}, \delta^{\prime \prime}, K^{\prime \prime}\right)
$$

from below and

$$
\psi\left(N^{\prime}, \delta^{\prime}, K^{\prime}\right) \cdot \psi\left(N^{\prime \prime}, \delta^{\prime \prime}, K^{\prime \prime}\right)
$$

from above, where $N^{\prime}, N^{\prime \prime}, \delta^{\prime}, \delta^{\prime \prime}, K^{\prime}, K^{\prime \prime}$ satisfy (3.4)-(3.7). Hence for $N>>0$, by (5.10),

$$
\begin{aligned}
N \geq N^{\prime} N^{\prime \prime} & >N\left(\frac{\delta}{\log N}\right)^{40}>N^{\frac{99}{100}} \\
N^{\prime}+N^{\prime \prime} & <\left(\frac{K}{\delta}\right)^{15} N^{1 / 2}<N^{\frac{11}{20}} \\
\delta^{\prime} \delta^{\prime \prime} & >\frac{10^{36} \delta}{(\log N)^{6}} \\
K^{\prime} K^{\prime \prime} & <\delta^{-14}(\log N)^{10} K .
\end{aligned}
$$

Condition (5.14) indeed reduces $N$ to scale $N^{\frac{11}{20}}$ for which we assume (5.1), (5.2) admissible (notice that since $N>\bar{N}, \log N^{\prime}, \log N^{\prime \prime} \gtrsim \log \bar{N}$ ). In what follows, the role of the additional technical factors in (5.1), (5.2) will become apparent.

Substituting (5.1) in (5.11), we get

$$
\left(K^{\prime} K^{\prime \prime}\right)^{-A_{1}}\left(\delta^{\prime}\right)^{A_{2} \ell \ell N^{\prime}}\left(\delta^{\prime \prime}\right)^{A_{2} \ell \ell N^{\prime \prime}} e^{A_{3}\left[\left(\ell \ell N^{\prime}\right)^{2}+\left(\ell \ell N^{\prime \prime}\right)^{2}\right]}\left(N^{\prime} N^{\prime \prime}\right)^{1-\tau} .
$$

From (5.13), (5.14)

$$
\begin{aligned}
N^{\frac{11}{25}} & <N^{\prime}, N^{\prime \prime}<N^{\frac{11}{20}}, \\
\frac{99}{100} \ell \ell N & <\ell \ell N-\log \frac{25}{11}<\ell \ell N^{\prime}, \quad \ell \ell N^{\prime \prime}<\ell \ell N-\log \frac{20}{11} .
\end{aligned}
$$

From (5.13)-(5.16), (5.18), the expression in (5.17) is at least

$$
\begin{aligned}
& \delta^{14 A_{1}}(\log N)^{-10 A_{1}} K^{-A_{1}}\left[\frac{\delta}{(\log N)^{6}}\right]^{A_{2}\left(\ell \ell N-\log \frac{20}{11}\right)} e^{\frac{19}{10} A_{3}(\ell \ell N)^{2}} N^{1-\tau}\left(\frac{\delta}{\log N}\right)^{40(1-\tau)} \\
& >K^{-A_{1}} \cdot \delta^{A_{2} \ell \ell N} e^{A_{3}(\ell \ell N)^{2}} N^{1-\tau} \cdot u \cdot v,
\end{aligned}
$$


where

$$
\begin{aligned}
& u=(\log N)^{-10 A_{1}-6 A_{2} \ell \ell N-40} e^{\frac{9}{10} A_{3}(\ell \ell N)^{2}}, \\
& v=\delta^{14 A_{1}-\left(\log \frac{20}{11}\right) A_{2}+40}
\end{aligned}
$$

and each of the factors (5.19) and (5.20) will be at least 1 for suitable choices $A_{1}<A_{2}<A_{3}\left(A_{1} \sim A_{2} \sim A_{3}\right)$. Hence (5.17) still admits (5.1) as lower bound.

Similarly, substituting (5.2) in (5.12), we get

$$
\begin{aligned}
& \left(K^{\prime} K^{\prime \prime}\right)^{B_{1}}\left(\delta^{\prime}\right)^{-B_{2} \ell \ell N^{\prime}}\left(\delta^{\prime \prime}\right)^{-B_{2} \ell \ell N^{\prime \prime}} e^{-B_{3}\left[\left(\ell \ell N^{\prime}\right)^{2}+\left(\ell \ell N^{\prime \prime}\right)^{2}\right]}\left(N^{\prime} N^{\prime \prime}\right)^{\gamma} \\
& \quad<\delta^{-14 B_{1}}(\log N)^{10 B_{1}} K^{B_{1}}\left[\frac{(\log N)^{6}}{\delta}\right]^{B_{2}\left(\ell \ell N-\log \frac{20}{11}\right)} e^{-\frac{19}{10} B_{3}(\ell \ell N)^{2}} N^{\gamma} \\
& \quad<K^{B_{1}} \cdot \delta^{-B_{2} \ell \ell N} e^{-B_{3}(\ell \ell N)^{2}} N^{\gamma} \cdot u^{\prime} \cdot v^{\prime},
\end{aligned}
$$

where

$$
\begin{aligned}
& u^{\prime}=(\log N)^{10 B_{1}+6 B_{2} \ell \ell N} \cdot e^{-\frac{9}{10} B_{3}(\ell \ell N)^{2}}, \\
& v^{\prime}=\delta^{-14 B_{1}+B_{2} \log \frac{20}{11}} .
\end{aligned}
$$

Again a choice of $B_{3}>B_{2}>B_{1}\left(B_{3} \sim B_{2} \sim B_{1}\right)$ allows us to get (5.21) and (5.22) at most 1.

Thus (5.12) still satisfies (5.2).

This proves Lemma 5.1 .

Conclusion of the proof of Proposition 3. Immediate from Lemma 5.1.

First, we may assume $K=K(\mathcal{G})<N^{\frac{1}{\Lambda}}$ since (2.2) is otherwise obvious.

Apply Lemma 5.1 with $\tau, \gamma$ replaced by $\frac{\tau}{2}, \frac{\gamma}{2}$ and let

$$
\Lambda=\frac{2 A_{1}}{\tau}+A_{2}+B_{1}+\frac{2 B_{2}}{\gamma} .
$$

The choice of $\Lambda$ implies that $\Lambda>\frac{2 A_{1}}{\tau}, \Lambda>B_{1}, A_{2}$, and $\frac{\Lambda}{2 B_{2}} \gamma>1$. In (5.1)

$$
\phi(N, \delta, K)>\delta^{A_{2} \ell \ell N} N^{1-\frac{\tau}{2}-\frac{A_{1}}{\Lambda}}>\delta^{\Lambda \ell \ell N} N^{1-\tau} .
$$

In $(5.2)$

$$
\psi(N, \delta, K)<K^{\Lambda} \cdot \delta^{-B_{2} \ell \ell N} N^{\frac{\gamma}{2}} .
$$

If $\delta^{-B_{2} \ell \ell N}<N^{\frac{\gamma}{2}}$, then $\psi(N, \delta, K)<K^{\Lambda} N^{\gamma}$. Otherwise in (2.1)

$$
\delta^{\Lambda \ell \ell N} N^{1-\tau}<N^{-\frac{\Lambda}{2 B_{2}} \gamma} N^{1-\tau}<1
$$

hence the statement becomes trivial.

\section{REMARKS}

(1) Going back to (1.12)-(1.15) and the proofs of Lemma 5.1 and Proposition 3, an inspection of the argument shows that one may take $k(b)=C^{b^{4}}$ in the theorem (for some constant $C$ ). We certainly did not try to proceed efficiently here.

(2) The proof of the theorem shows in fact the following stronger statement:

For all $b \in \mathbb{Z}_{+}, \delta>0$, there is $k=k(b, \delta) \in \mathbb{Z}_{+}$such that whenever $A \subset \mathbb{Z}$, $|A|=N$ sufficiently large, then either $\left|A^{(k)}\right|>N^{b}$ or $\left|h A_{1}\right|>\left|A_{1}\right|^{h-\delta}$ for all $A_{1} \subset A,\left|A_{1}\right|>N^{\delta}$ and $h \leq b$. 
(3) As in [Ch], our approach uses strongly prime factorization in $\mathbb{Z}$. Thus the argument at this point does not apply to subsets $A \subset \mathbb{R}$.

\section{REFERENCES}

[B-K] J. Bourgain, S. Konjagin, Estimates for the number of sums and products and for exponential sums over subgroups in fields of prime order, C. R. Acad. Sci. Paris Ser. I 337 (2003), 75-80.

[Ch] M. Chang, Erdös- Szemerédi sum-product problem, Annals of Math. 157 (2003), 939-957.

[E] G. Elekes, On the number of sums and products, Acta Arithmetica 81, Fase 4 (1997), 365-367. MR 98h:11026

[E-N-R] G. Elekes, M. Nathanson, I. Rusza, Convexity and sumsets, J. Number Theory 83 (2000), 194-201. MR 2001e:11020

[E-S] P. Erdős, E. Szemerédi, On sums and products of integers, in P. Erdös, L. Alpár, G. Halász (editors), Studies in Pure Mathematics; in memory of P. Turán, pp. 213-218. MR 86m:11011

[Go] W. T. Gowers, A new proof of Szemerédi's theorem for arithmetic progressions of length 4, GAFA 8 (1998), 529-551. MR 2000d:11019

[Ki] S. V. Kisliakov, Banach Spaces and Classical Harmonic Analysis, in Handbook for the geometry of Banach Spaces,Vol. 1, pp. 871-898, North-Holland, Amsterdam, 2001. MR 2003d:46021

[K] S. Konjagin, Private communication.

[Na] M. B. Nathanson, Additive Number Theory: Inverse Problems and the Geometry of Sumsets, Springer, 1996. MR 98f:11011

[Ru] W. Rudin, Trigonometric series with gaps, J. Math. Mech. 9 (1960), 203-227.

[So] J. Solymosi, On the number of sums and products, preprint, 2003.

Institute for Advanced Study, Olden Lane, Princeton, New Jersey 08540

E-mail address: bourgain@math.ias.edu

Mathematics Department, University of California, Riverside, California 92521

E-mail address: mcc@math.ucr.edu 